TRANSACTIONS OF THE

AMERICAN MATHEMATICAL SOCIETY

Volume 356, Number 5, Pages 1705-1726

S 0002-9947(04)03542-1

Article electronically published on January 6, 2004

\title{
ROOT VECTORS FOR GEOMETRICALLY SIMPLE TWO-PARAMETER EIGENVALUES
}

\author{
PAUL BINDING AND TOMAŽ KOŠIR
}

\begin{abstract}
A class of two-parameter eigenvalue problems involving generally nonselfadjoint and unbounded operators is studied. A basis for the root subspace at a geometrically simple eigenvalue of Fredholm type is computed in terms of the underlying two-parameter system. Comparison with Faierman's work on two-parameter boundary value problems of Sturm-Liouville type is given as an application.
\end{abstract}

\section{INTRODUCTION}

We consider a pair of two-parameter equations of the form

$$
W_{i}(\boldsymbol{\lambda}) x_{i}=0 \neq x_{i},
$$

where

$$
W_{i}(\boldsymbol{\lambda})=A_{i 0} \lambda_{0}+A_{i 1} \lambda_{1}+A_{i 2},
$$

$A_{i j}(j=1,2)$ are bounded linear operators acting on Hilbert spaces $H_{i}(i=1,2)$ over the complex numbers, $A_{i 0}$ are closed densely defined with domain $\mathcal{D}\left(A_{i 0}\right) \subseteq$ $H_{i}$, and $\boldsymbol{\lambda}=\left(\lambda_{0}, \lambda_{1}\right) \in \mathbb{C}^{2}$. Evidently, $W_{i}(\boldsymbol{\lambda})$ is a linear operator with domain $\mathcal{D}\left(A_{i 0}\right)$ for all such parameters $\boldsymbol{\lambda}$.

The spectral theory of such systems has been developed from various viewpoints; see [1, 8, 22, 24, 26] for books on the subject. In most of the literature, the $A_{i j}$ are selfadjoint and satisfy definiteness conditions guaranteeing that the eigenvalues are simple in certain senses. A standard selfadjoint example is when (1.1) are Sturm-Liouville equations. One application of these ideas is to completeness and expansion theory of elliptic boundary value problems via separation of variables. For work on nonselfadjoint problems we refer to [5, 9, 11, 14, 15].

To introduce our analysis, we make the following constructions. The operators $A_{i j}, j=1,2$, induce operators $A_{i j}^{\dagger}$ on the Hilbert space tensor product $H=H_{1} \otimes H_{2}$ by means of

$$
A_{1 j}^{\dagger}\left(x_{1} \otimes x_{2}\right)=A_{1 j} x_{1} \otimes x_{2} \text { and } A_{2 j}^{\dagger}\left(x_{1} \otimes x_{2}\right)=x_{1} \otimes A_{2 j} x_{2}
$$

on decomposable tensors, extended by linearity and continuity to the whole of $H$. Similarly, $A_{i 0}$ induces an operator $A_{i 0}^{\dagger}$ with domain $\mathcal{D}\left(A_{i 0}^{\dagger}\right) \subset H$ (see [24, §2.3]).

Received by the editors June 15, 2001.

2000 Mathematics Subject Classification. Primary 35P10, 47A13; Secondary 35J55.

The first author's research was supported by NSERC of Canada.

The second author's research was supported by the Ministry of Science and Technology of Slovenia. 
We denote by $\mathcal{D}$ the intersection $\mathcal{D}\left(A_{10}^{\dagger}\right) \cap \mathcal{D}\left(A_{20}^{\dagger}\right)$, which is a dense subspace of $H$. We define the operator

$$
\Delta_{0}=\left|\begin{array}{ll}
A_{11}^{\dagger} & A_{12}^{\dagger} \\
A_{21}^{\dagger} & A_{22}^{\dagger}
\end{array}\right|=A_{11} \otimes A_{22}-A_{12} \otimes A_{21}
$$

on $H$ and operators

$$
\Delta_{1}=-A_{22}^{\dagger} A_{10}^{\dagger}+A_{12}^{\dagger} A_{20}^{\dagger} \text { and } \Delta_{2}=-A_{11}^{\dagger} A_{20}^{\dagger}+A_{21}^{\dagger} A_{10}^{\dagger}
$$

on $\mathcal{D}$. In $\S 2$ we shall make assumptions guaranteeing the existence and commutativity of the operators $\Gamma_{j}=\Delta_{2}^{-1} \Delta_{j}, j=0,1$, on $\mathcal{D}$.

The nullspace of an operator $A$ is denoted by $\mathcal{N}(A)$. A pair $\boldsymbol{\lambda}=\left(\lambda_{0}, \lambda_{1}\right) \in \mathbb{C}^{2}$ is called an eigenvalue of the pair of commuting linear transformations $\left\{\Gamma_{0}, \Gamma_{1}\right\}$ if

$$
\mathcal{R}_{1}=\mathcal{R}_{1}(\boldsymbol{\lambda})=\mathcal{N}\left(\Gamma_{0}-\lambda_{0} I\right) \cap \mathcal{N}\left(\Gamma_{1}-\lambda_{1} I\right) \neq\{0\} .
$$

The subspace $\mathcal{R}_{1}$ is called the geometric eigenspace corresponding to $\boldsymbol{\lambda}$. The set of all the eigenvalues is called the point spectrum of $\left\{\Gamma_{0}, \Gamma_{1}\right\}$. We say that $\boldsymbol{\lambda}$ is a geometrically simple eigenvalue of $\left\{\Gamma_{0}, \Gamma_{1}\right\}$ if $\operatorname{dim} \mathcal{R}_{1}=1$. Under assumptions to be specified in $\S 2$, the eigenvalues $\boldsymbol{\lambda}$ of (1.2) and that of its associated linear transformations $\Gamma_{0}, \Gamma_{1}$ coincide and we have

$$
\mathcal{R}_{1}(\boldsymbol{\lambda})=\mathcal{N}\left(W_{1}(\boldsymbol{\lambda})\right) \otimes \mathcal{N}\left(W_{2}(\boldsymbol{\lambda})\right) .
$$

(See [5, §4].) We shall simply refer to such $\boldsymbol{\lambda}$ as eigenvalues from now on.

We define the $m$-th root subspace

$$
\mathcal{R}_{m}=\mathcal{R}_{m}(\boldsymbol{\lambda})=\bigcap_{k=0}^{m} \mathcal{N}\left[\left(\Gamma_{0}-\lambda_{0} I\right)^{k}\left(\Gamma_{1}-\lambda_{1} I\right)^{m-k}\right],
$$

for $m \geq 2$. The least integer $M$, if it exists, such that $\mathcal{R}_{M}=\mathcal{R}_{M+1}$ is called the ascent of $\boldsymbol{\lambda}$. We call the subspace

$$
\mathcal{R}=\mathcal{R}(\boldsymbol{\lambda})=\bigcup_{m=1}^{\infty} \mathcal{R}_{m}
$$

the root subspace of $\mathbf{W}=\left(W_{1}, W_{2}\right)$ at $\boldsymbol{\lambda}$. Note that each subspace $\mathcal{R}_{m}$ is an invariant subpace for both $\Gamma_{0}$ and $\Gamma_{1}$. If the ascent of $\boldsymbol{\lambda}$ is equal to 1 , then $\boldsymbol{\lambda}$ is called semisimple. If $\boldsymbol{\lambda}$ is a geometrically simple eigenvalue and its ascent is equal to 1 , then $\mathcal{R}=\mathcal{R}_{1}$ is one-dimensional and $\boldsymbol{\lambda}$ is called algebraically simple.

The elements of $\mathcal{R}$ are called root vectors and it is our purpose to describe bases of root vectors for $\mathcal{R}$ in terms of the $A_{i j}$. This problem was raised by Atkinson 1] and has been studied in certain definite selfadjoint cases by many authors. See [26] for a review of eigenvector completeness under various conditions including positive definiteness of $\Delta_{2}$. In these cases the eigenvalues are real (i.e. $\boldsymbol{\lambda} \in \mathbb{R}^{2}$ ) and always $\operatorname{dim} \mathcal{R}=\operatorname{dim} \mathcal{R}_{1}$. Thus if an eigenvalue is geometrically simple, then it is also algebraically simple. Moreover, (1.6) shows that a basis for $\mathcal{R}=\mathcal{R}_{1}$ can be constructed from the eigenvectors $x_{1} \otimes x_{2}$ for the $\Gamma_{j}, x_{i}$ as in (1.1).

Without definiteness, however, this may fail and various authors have tackled Atkinson's question. We cite [3] on real eigenvalues for selfadjoint problems and [8] on general eigenvalues for Sturm-Liouville (and therefore geometrically simple) problems. Faierman gives bases of $\mathcal{R}_{l}$ for $l=2$ and 3 and gives a conjecture for general $l$. In an earlier paper [5] we gave a basis for $\mathcal{R}_{2}$ for general nonselfadjoint 
(including geometrically nonsimple) problems. A coalgebraic approach to this problem in [11] describes root subspaces $\mathcal{R}_{l}$ for all $l$ in terms of $A_{i j}$, but it is difficult to use it to construct bases explicitly. Here we shall give a general construction for a basis of $\mathcal{R}$ for geometrically simple eigenvalues of problems that are not necessarily selfadjoint. We shall relate our construction in the Sturm-Liouville case to Faierman's work in $\S 5$.

Our plan is as follows. Our main assumptions are discussed in $\S 2$, followed by the construction of a basis for the root subspace in the so-called nonderogatory case. In $\S 3$, we lay foundations for the more difficult derogatory case. The main result (Theorem 4.1) is proved by induction on $m$ (see (1.7) $)$ in $\S 4$. $\S 5$ contains the specialization to the Sturm-Liouville case.

\section{Preliminaries}

We begin with our assumptions on the $A_{i j}$. We make two regularity assumptions and a solvability assumption.

Assumption I. There exists $\alpha \in \mathbb{C}$ such that the operator $\Delta_{2}^{\prime}=\Delta_{2}+\alpha \Delta_{0}: \mathcal{D} \rightarrow$ $H$ has a bounded inverse.

This assumption holds in many interesting cases. We refer to [5, p. 231] for a discussion.

To simplify the notation we assume, unless stated otherwise, that the operator $\Delta_{2}$ has a bounded inverse. This form of Assumption I can be obtained by a shift in parameters.

Let $\mathcal{A}_{i j}^{\dagger}$ denote the restriction of $A_{i j}^{\dagger}$ to $\mathcal{D}$. The array

$$
\mathcal{A}=\left[\begin{array}{lll}
\mathcal{A}_{10}^{\dagger} & \mathcal{A}_{11}^{\dagger} & \mathcal{A}_{12}^{\dagger} \\
\mathcal{A}_{20}^{\dagger} & \mathcal{A}_{21}^{\dagger} & \mathcal{A}_{22}^{\dagger}
\end{array}\right]
$$

then defines a linear map $\mathcal{A}: \mathcal{D}^{3} \longrightarrow H^{2}$. Here $H^{k}$ is the direct sum of $k$ copies of $H$. Omitting the $j$-th column we get a transformation $\mathcal{A}_{j}$ acting on the (algebraic) direct sum $\mathcal{D}^{2}$ for $j=0,1,2$. Note that $\Delta_{j}=(-1)^{j} \operatorname{det} \mathcal{A}_{j}$. We denote by $\mathcal{C}_{j}$ the $j$-th column of $\mathcal{A}$. Now we state the solvability assumption.

Assumption II. The equation $\mathcal{A}_{2} \mathbf{y}=\mathcal{C}_{2} x$ has a solution $\mathbf{y} \in \mathcal{D}^{2}$ for all $x \in \mathcal{D}$.

Again see [5] p. 236] for a discussion of this assumption.

The linear transformations $\Gamma_{j}=\Delta_{2}^{-1} \Delta_{j},(j=0,1)$ act on $\mathcal{D}$. These are called the associated linear transformations of the two-parameter system (1.2). Under Assumption II it follows from [5, Thm. 3.2] that the linear transformations $\Gamma_{0}$ and $\Gamma_{1}$ commute on $\mathcal{D}$, i.e., $\Gamma_{0} \Gamma_{1} x=\Gamma_{1} \Gamma_{0} x$ for all $x \in \mathcal{D}$, and that

$$
A_{i 0}^{\dagger} \Gamma_{0} x+A_{i 1}^{\dagger} \Gamma_{1} x+A_{i 2}^{\dagger} x=0
$$

for $i=1,2$ and all $x \in \mathcal{D}$. Relation (2.1) is a consequence of Assumption II that will be often used in the proofs.

Next we define the notions of eigenvalues and point spectra for a two-parameter system. A point $\boldsymbol{\lambda} \in \mathbb{C}^{2}$ is called an eigenvalue of the two-parameter system (1.2) if both nullspaces $\mathcal{N}\left(W_{i}(\boldsymbol{\lambda})\right), i=1,2$, are nontrivial. The set of all eigenvalues is called the point spectrum of (1.2), and it is denoted by $\sigma(\mathbf{W})$. An eigenvalue $\boldsymbol{\lambda} \in \sigma(\mathbf{W})$ is called geometrically simple if $\operatorname{dim} \mathcal{N}\left(W_{i}(\boldsymbol{\lambda})\right)=1$ for both $i$. Thus $\boldsymbol{\lambda}$ is a geometrically simple eigenvalue of (1.2) if and only if it is a geometrically 
simple eigenvalue for the pair of associated linear transformations $\left(\Gamma_{0}, \Gamma_{1}\right)$ although perhaps not for either of $\Gamma_{j}$ separately.

Assumption III. $\boldsymbol{\lambda}=\left(\lambda_{0}, \lambda_{1}\right)$ is a geometrically simple eigenvalue of finite ascent $M$ and $W_{i}(\boldsymbol{\lambda}), i=1,2$, are Fredholm of index 0 [25].

This assumption is satisfied, for example, in several cases arising from boundary value problems, e.g., of Sturm-Liouville type (see §5). Assumption III is in terms of the maps $A_{i j}$ except for the assumption of finite ascent. This is defined in terms of the associated linear transformations. It follows from our results in $\S 4$ that an equivalent definition of the ascent is possible in terms of the $A_{i j}$ - it is the least $m$ such that $\mathcal{N}\left(\mathcal{S}_{m}\right)=0$.

In view of Assumption III we have $\operatorname{dim} \mathcal{N}\left(W_{i}(\boldsymbol{\lambda})\right)=\operatorname{dim} \mathcal{N}\left(W_{i}(\boldsymbol{\lambda})^{*}\right)=1$ for

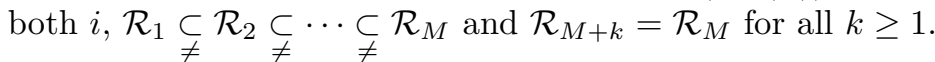

Finally, we remark that Assumptions I-III of our present setup are stronger than Assumptions I-III of [5] or [21] in the case $n=2$. Assumption I here and in [5] 21] is the same. Here and in [21] we use the same solvability assumption while in [5] a weaker solvability assumption is used. In [21] it is assumed further that $\boldsymbol{\lambda}$ is geometrically simple. In both [5, 21] it is assumed that the $W_{i}(\boldsymbol{\lambda})$ are Fredholm operators not necessarily of index 0 . The assumption of Fredholm index 0 here is made simply for ease of notation.

Next we state two-parameter versions of some results of [5, 18, 19, 20] on root subspaces at geometrically simple eigenvalues of commuting linear maps and of multiparameter systems. These versions are used in what follows.

We write

for $m \geq 1$. Here $D_{0}=0$.

$$
D_{m}=\operatorname{dim} \mathcal{R}_{m} \text { and } d_{m}=D_{m}-D_{m-1}
$$

Lemma 2.1. If $\boldsymbol{\lambda}$ is a geometrically simple eigenvalue, then for each $m \geq 2$ the subspace $\mathcal{R}_{m}$ is finite-dimensional.

Proof. The argument is similar to the one used in the proof of [5, Lemma 5.2]. It is by induction on $m$. Assume $m \geq 2$. The subspace $\mathcal{R}_{m}$ is invariant for both $\Gamma_{j}-\lambda_{j} I, j=0,1$. The range $\mathcal{R}\left(\left.\left(\Gamma_{j}-\lambda_{j} I\right)\right|_{\mathcal{R}_{m}}\right)$ is contained in $\mathcal{R}_{m-1}$ and thus is finite-dimensional because of the inductive assumption. Each kernel $\mathcal{N}\left(\left.\left(\Gamma_{j}-\lambda_{j} I\right)\right|_{\mathcal{R}_{m}}\right)$ has finite codimension in $\mathcal{R}_{m}$, i.e., the orthogonal complement $\mathcal{Q}_{j}$ of $\mathcal{N}\left(\left.\left(\Gamma_{j}-\lambda_{j} I\right)\right|_{\mathcal{R}_{m}}\right)$ in $\mathcal{R}_{m}$ is finite-dimensional. Hence the linear span $\mathcal{Q}$ of the $\mathcal{Q}_{j}, j=0,1$, is finite-dimensional. Then $\mathcal{R}_{m}=\mathcal{R}_{m-1}+\mathcal{Q}$ also has finite dimension.

Since $\boldsymbol{\lambda}$ is geometrically simple, (1.6) implies that $d_{1}=1$. The restricted maps of $\Gamma_{0}$ and $\Gamma_{1}$ to $\mathcal{R}_{m}$ commute, and so it follows that $d_{i} \leq i$ for all $i \geq 1$. Suppose that

$$
x_{i 1} \in \mathcal{N}\left(W_{i}(\boldsymbol{\lambda})\right), \quad i=1,2,
$$

are nonzero vectors. Then the vector

$$
z_{1}=x_{11} \otimes x_{21}
$$

spans $\mathcal{R}_{1}$. Further, we choose nonzero vectors $y_{i 1} \in \mathcal{N}\left(W_{i}(\boldsymbol{\lambda})^{*}\right)(i=1,2)$ and we write

$$
\mathcal{S}_{2}=\left[\begin{array}{ll}
\left\langle A_{10} x_{11}, y_{11}\right\rangle & \left\langle A_{11} x_{11}, y_{11}\right\rangle \\
\left\langle A_{20} x_{21}, y_{21}\right\rangle & \left\langle A_{21} x_{21}, y_{21}\right\rangle
\end{array}\right]
$$


Here we use $\langle x, y\rangle$ to denote the scalar product of vectors $x, y$. It is clear from the context which Hilbert space $H_{i}$ or $H$ is meant. Now [5, Cor. 6.4] states that $d_{2}=\operatorname{dim} \mathcal{N}\left(\mathcal{S}_{2}\right)$. If $d_{2} \leq 1$, then we call $\boldsymbol{\lambda}$ nonderogatory. Otherwise, $d_{2}=2$, and we call $\boldsymbol{\lambda}$ derogatory. Bases for the root subspaces $\mathcal{R}_{m}$ corresponding to a nonderogatory eigenvalue of an $n$-parameter system (for $n \geq 2$ ) are constructed in [19] in the finite-dimensional setup. For completeness we will give an infinitedimensional two-parameter version of the main result of [19] (see Theorem 2.2 below).

For $\mathbf{c}=\left[c_{1}, c_{2}\right]^{T} \in \mathbb{C}^{2}$ and $i=1,2$, we write

$$
U_{i}(\mathbf{c})=A_{i 0} c_{1}+A_{i 1} c_{2} .
$$

If $M=1$, then $z_{1}$ spans $\mathcal{R}_{M}$. Assume from now on that $M \geq 2$. The following is a two-parameter analogue of the main result of [19]. It gives a basis for the root subspace at a nonderogatory eigenvalue.

Theorem 2.2. Suppose that $\boldsymbol{\lambda} \in \mathbb{C}^{2}$ is a nonderogatory eigenvalue for a twoparameter system (1.2) and suppose that $2 \leq m \leq M$. Then there exist $\mathbf{c}_{2}, \mathbf{c}_{3}, \ldots, \mathbf{c}_{m}$ $\in \mathbb{C}^{2}, \mathbf{c}_{2} \neq 0$, and $x_{i 2}, x_{i 3}, \ldots, x_{i m} \in \mathcal{D}\left(A_{i 0}\right), i=1,2$, such that

$$
\sum_{j=1}^{k-1} U_{i}\left(\mathbf{c}_{k+1-j}\right) x_{i j}+W_{i}(\boldsymbol{\lambda}) x_{i k}=0 \quad \text { for } k=2,3, \ldots, m, i=1,2 .
$$

Moreover, for $k=1,2, \ldots, m$, the vectors

$$
z_{k}=\sum_{j=1}^{k} x_{1 j} \otimes x_{2, k+1-j}
$$

are such that

$$
\left(\Gamma_{i-1}-\lambda_{i-1} I\right) z_{k}=\sum_{j=1}^{k-1} c_{k+1-j, i} z_{j}, \quad i=1,2,
$$

where $\mathbf{c}_{k}=\left[\begin{array}{ll}c_{k 1} & c_{k 2}\end{array}\right]^{T}$ and $\left\{z_{1}, z_{2}, \ldots, z_{m}\right\}$ is a basis for the $m$-th root subspace $\mathcal{R}_{m}$.

Proof. The theorem is proved by induction on $m$. For each $m$ we choose a vector $z_{m} \in \mathcal{R}_{m} \backslash \mathcal{R}_{m-1}$ and use Assumptions II and III to prove the existence of vectors $x_{i j} \in \mathcal{D}\left(A_{i 0}\right)$ such that (2.6) and (2.7) hold. Here the arguments follow closely those in the proof of [5, Thm. 6.2]. For the converse, (2.7) follows from (2.5) and (2.6) by a direct calculation (see [19, Lemma 13]).

\section{Derogatory eigenvalues}

We assume now that $\boldsymbol{\lambda}$ is a geometrically simple eigenvalue which is derogatory, i.e., $\mathcal{S}_{2}=0$ or equivalently $d_{2}=2$. First we recall some results on the structure of nilpotent commutative matrices with one-dimensional joint kernel and $d_{2}=2$ (see $[18,[20])$. 
3.1. Structure of nilpotent commutative matrices. We denote by $\mathcal{R}=\mathcal{R}_{M}$ the root subspace corresponding to $\boldsymbol{\lambda}$ and we write $N=\operatorname{dim} \mathcal{R}$. The restricted linear maps $C_{i}=\left.\left(\Gamma_{i}-\lambda_{i} I\right)\right|_{\mathcal{R}}, i=0,1$, are commuting and nilpotent. There exists a basis

$$
\mathcal{B}=\left\{z_{1}, z_{21}, z_{22}, z_{31}, \ldots, z_{3 d_{3}}, \ldots, z_{M 1}, z_{M 2}, \ldots, z_{M d_{M}}\right\}
$$

for $\mathcal{R}$ such that for $m=1,2, \ldots, M$, the set

$$
\mathcal{B}_{m}=\left\{z_{1}, z_{21}, z_{22}, z_{31}, \ldots, z_{3 d_{3}}, \ldots, z_{m 1}, z_{m 2}, \ldots, z_{m d_{m}}\right\}
$$

is a basis for $\mathcal{R}_{m}$. We call such a basis $\mathcal{B}$ a filtered basis $($ for $\mathcal{R})$. In a filtered basis the matrices for $\mathbf{C}=\left\{C_{0}, C_{1}\right\}$ are simultaneously reduced to a special uppertriangular form and we view them as a cubic array of dimensions $N \times N \times 2$

$$
\mathbf{C}=\left[\begin{array}{ccccc}
\mathbf{0} & \mathbf{C}^{12} & \mathbf{C}^{13} & \ldots & \mathbf{C}^{1, M} \\
\mathbf{0} & \mathbf{0} & \mathbf{C}^{23} & \ldots & \mathbf{C}^{2, M} \\
\vdots & \vdots & \ddots & \ddots & \vdots \\
\mathbf{0} & \mathbf{0} & \mathbf{0} & \cdots & \mathbf{C}^{M-1, M} \\
\mathbf{0} & \mathbf{0} & \mathbf{0} & \cdots & \mathbf{0}
\end{array}\right]
$$

where

$$
\mathbf{C}^{k m}=\left[\begin{array}{cccc}
\mathbf{c}_{11}^{k m} & \mathbf{c}_{12}^{k m} & \cdots & \mathbf{c}_{1, d_{m}}^{k m} \\
\mathbf{c}_{21}^{k m} & \mathbf{c}_{22}^{k m} & \cdots & \mathbf{c}_{2, d_{m}}^{k m} \\
\vdots & \vdots & & \vdots \\
\mathbf{c}_{d_{k}, 1}^{k m} & \mathbf{c}_{d_{k}, 2}^{k m} & \cdots & \mathbf{c}_{d_{k}, d_{m}}^{k m}
\end{array}\right]
$$

is a cubic array of dimensions $d_{k} \times d_{m} \times 2$ and $\mathbf{c}_{g f}^{k m}=\left[\begin{array}{ll}c_{1 g f}^{k m} & c_{2 g f}^{k m}\end{array}\right]^{T} \in \mathbb{C}^{2}$. The column cross-sections

$$
\mathcal{C}_{f}^{k m}=\left[\begin{array}{llll}
\mathbf{c}_{1 f}^{k m} & \mathbf{c}_{2 f}^{k m} & \cdots & \mathbf{c}_{d_{k}, f}^{k m}
\end{array}\right], f=1,2, \ldots, d_{m},
$$

of $\mathbf{C}^{k m}$ are $2 \times d_{k}$ matrices. The constants $c_{i g f}^{k m}$ are determined by the relations

$$
C_{i-1} z_{m f}=\sum_{k=1}^{m-1} \sum_{g=1}^{d_{k}} c_{i g f}^{k m} z_{k g}, \quad i=1,2,
$$

where we write $z_{11}=z_{1}$. (For further details see [18, 20].)

For $m \geq 4$ we denote by $\Phi_{m}$ the set of multi-indices

$$
\left\{\left(l_{1}, l_{2}, l_{3}\right) ; l_{j} \geq 2, \sum_{j=1}^{3} l_{j} \leq m+2\right\},
$$

and for $\mathbf{l}=\left(l_{1}, l_{2}, l_{3}\right) \in \Phi_{m}$ we denote by $\chi_{\mathbf{l}}$ the set of multi-indices

$$
\left\{\left(h_{1}, h_{2}, h_{3}\right) ; 1 \leq h_{j} \leq d_{l_{j}} \text { for } j=1,2,3\right\} .
$$

We call a matrix $C$ symmetric if $C=C^{T}$.

For use in our proofs we state a version of some of results of [18, 20] for pairs of commutative matrices. Since $\mathbf{c}_{11}^{12}$ and $\mathbf{c}_{12}^{12}$ are linearly independent we can choose a basis for $\mathcal{R}_{2}$ such that $C_{i-1} z_{2 j}=\delta_{i j} z_{1}$, where $\delta_{i j}$ is the Kronecker symbol. By adding appropriate linear combinations of $z_{21}$ and $z_{22}$ to other vectors $z_{m f}, m \geq 3$, we find a basis $\mathcal{B}$ such that the following result holds. 
Theorem 3.1. There exists a filtered basis $\mathcal{B}$ for $\mathcal{R}$ and associated with it a set of symmetric matrices

$$
C_{f}^{m}=\left[C_{f}^{k l m}\right]_{k, l=2}^{m-1}, m=3,4, \ldots, M, f=1,2, \ldots, d_{m},
$$

where $C_{f}^{k l m}=\left[c_{g h f}^{k l m}\right]_{g=1, h=1}^{d_{k} \quad d_{l}}$ are $d_{k} \times d_{l}$ matrices, such that for the corresponding commutative array (3.2) we have:

(a) $\mathcal{C}_{1}^{12}=\left[\begin{array}{ll}1 & 0\end{array}\right]^{T}, \mathcal{C}_{2}^{12}=\left[\begin{array}{ll}0 & 1\end{array}\right]^{T}$ and $\mathcal{C}_{f}^{1 m}=\left[\begin{array}{ll}0 & 0\end{array}\right]^{T}$ for $m=3,4, \ldots$, $M$ and $f=1,2, \ldots, d_{m}$,

(b) $C_{f}^{2 k m}=\mathcal{C}_{f}^{k m}$ are the column cross-sections (3.3),

(c) for $m=3,4, \ldots, M$ the matrices $C_{f}^{2, m-1, m}, f=1,2, \ldots, d_{m}$, are linearly independent,

(d) $C_{f}^{k l m}=0$ if $k+l>m+1$,

(e) for $m=4,5, \ldots, M$ and $f=1,2, \ldots, d_{m}$ the relations

$$
\sum_{k=l_{1}+l_{2}-1}^{m-l_{3}+1} \sum_{g=1}^{d_{k}} c_{h_{1} h_{2} g}^{l_{1} l_{2} k} c_{h_{3} g f}^{l_{3} k m}=\sum_{k=l_{2}+l_{3}-1}^{m-l_{1}+1} \sum_{g=1}^{d_{k}} c_{h_{1} h_{3} g}^{l_{1} l_{3} k} c_{h_{2} g f}^{l_{2} k m}
$$

hold for all $\mathbf{l}=\left(l_{1}, l_{2}, l_{3}\right) \in \Phi_{m}$ and $\left(h_{1}, h_{2}, h_{3}\right) \in \chi_{\mathbf{l}}$.

We remark that property (e) in the above theorem together with the symmetry of matrices $C_{f}^{m}$ is equivalent to the commutativity of the matrices $C_{0}$ and $C_{1}$ and that $c_{g h f}^{k l m}$ are the structure constants for multiplication in the commutative algebra generated by $C_{0}, C_{1}$ and the identity matrix (see [20]).

In the rest of the section we consider, unless stated otherwise, only filtered bases $\mathcal{B}$ such that the associated matrices $C_{m}^{f}$ satisfy the properties (a)-(e) of Theorem 3.1 .

3.2. Bases for $\mathcal{R}_{m}$ for $m=2,3$. We denote by $\mathcal{D}_{i}^{\prime}$ the (vector space) intersection $\mathcal{D}\left(A_{i 0}\right) \cap\left(\mathcal{N}\left(W_{i}(\boldsymbol{\lambda})\right)\right)^{\perp}$, where $\left(\mathcal{N}\left(W_{i}(\boldsymbol{\lambda})\right)\right)^{\perp}$ is the orthogonal complement of $\mathcal{N}\left(W_{i}(\boldsymbol{\lambda})\right)$ in $H_{i}$.

Lemma 3.2. For $i=1,2$, the subspace $\mathcal{D}_{i}^{\prime}$ is an infinite-dimensional vector subspace of $\mathcal{D}_{i}=\mathcal{D}_{i}\left(A_{i 0}\right)$.

Proof. Since the lattice of vector subspaces is modular [7, p. 13] and since $\mathcal{N}\left(W_{i}(\boldsymbol{\lambda})\right)$ $\subset \mathcal{D}_{i}$, it follows that

$$
\mathcal{D}_{i}=\left(\mathcal{N}\left(W_{i}(\boldsymbol{\lambda})\right) \oplus\left(\mathcal{N}\left(W_{i}(\boldsymbol{\lambda})\right)\right)^{\perp}\right) \cap \mathcal{D}_{i}=\mathcal{N}\left(W_{i}(\boldsymbol{\lambda})\right)+\left(\mathcal{N}\left(W_{i}(\boldsymbol{\lambda})\right)^{\perp} \cap \mathcal{D}_{i}\right) .
$$

Since $\mathcal{N}\left(W_{i}(\boldsymbol{\lambda})\right)$ is finite-dimensional and $\mathcal{D}_{i}$ infinite-dimensional, $\mathcal{D}_{i}^{\prime}$ is also an infinite-dimensional vector space.

The following is a technical observation, which enables us to shorten proofs but has no significance otherwise. In actual calculations of basis vectors we can choose vectors $x_{i k}^{g} \in \mathcal{D}\left(A_{i 0}\right)$ (i.e. not necessarily $\left.x_{i k}^{g} \in \mathcal{D}_{i}^{\prime}\right)$ that satisfy all other required conditions.

Lemma 3.3. Suppose that a vector $x_{i} \in\left(\mathcal{N}\left(W_{i}(\boldsymbol{\lambda})^{*}\right)\right)^{\perp}$. Then there exists a vector $v_{i} \in \mathcal{D}_{i}^{\prime}$ such that $x_{i}=W_{i}(\boldsymbol{\lambda}) v_{i}$. 
Proof. Suppose that $x_{i} \in\left(\mathcal{N}\left(W_{i}(\boldsymbol{\lambda})^{*}\right)\right)^{\perp}$. Since $W_{i}(\boldsymbol{\lambda})$ is a Fredholm operator, its range is a closed subspace of $H_{i}[25]$ and therefore $\left(\mathcal{N}\left(W_{i}(\boldsymbol{\lambda})^{*}\right)\right)^{\perp}=\mathcal{R}\left(W_{i}(\boldsymbol{\lambda})\right)$. Thus there exists a vector $u_{i} \in \mathcal{D}\left(A_{i 0}\right)$ such that $x_{i}=W_{i}(\boldsymbol{\lambda}) u_{i}$. Since $\mathcal{D}\left(W_{i}(\boldsymbol{\lambda})\right)=$ $\mathcal{D}\left(A_{i 0}\right)$ we can find vectors $v_{i} \in \mathcal{D}_{i}^{\prime}$ and $w_{i} \in \mathcal{N}\left(W_{i}(\boldsymbol{\lambda})\right)$ such that $v_{i}+w_{i}=u_{i}$. Then it follows that $x_{i}=W_{i}(\boldsymbol{\lambda}) v_{i}$.

The following result is a special case of [5 Thm. 6.3]. Note that the additional condition that $x_{i 2}^{k} \in \mathcal{D}_{i}^{\prime}$ is a consequence of Lemma 3.3

Theorem 3.4. Assume that $\boldsymbol{\lambda} \in \sigma(\mathbf{W})$ is a derogatory eigenvalue, i.e., $\mathcal{S}_{2}=0$, and that $\mathbf{c}_{11}^{12}=\left[\begin{array}{ll}1 & 0\end{array}\right]^{T}$ and $\mathbf{c}_{12}^{12}=\left[\begin{array}{ll}0 & 1\end{array}\right]^{T}$. Then there exist vectors $x_{i 2}^{k} \in \mathcal{D}_{i}^{\prime}$ such that

$$
U_{i}\left(\mathbf{c}_{1 k}^{12}\right) x_{i 1}+W_{i}(\boldsymbol{\lambda}) x_{i 2}^{k}=0
$$

for $i, k=1,2$. Furthermore, the vectors

$$
z_{2 k}=x_{11} \otimes x_{22}^{k}+x_{12}^{k} \otimes x_{21}
$$

are such that

$$
\left(\Gamma_{i-1}-\lambda_{i-1} I\right) z_{2 k}=\delta_{i k} z_{1}
$$

for $i, k=1,2$, and $\left\{z_{1}, z_{21}, z_{22}\right\}$ is a basis for $\mathcal{R}_{2}$. Here $\delta_{i k}$ is the Kronecker symbol.

Next we define the matrix $\mathcal{S}_{3}$ by

$$
\mathcal{S}_{3}=\left[\begin{array}{lll}
\left\langle A_{10} x_{12}^{1}, y_{11}\right\rangle & \left\langle A_{10} x_{12}^{2}, y_{11}\right\rangle+\left\langle A_{11} x_{12}^{1}, y_{11}\right\rangle & \left\langle A_{11} x_{12}^{2}, y_{11}\right\rangle \\
\left.A_{20} x_{22}^{1}, y_{21}\right\rangle & \left\langle A_{20} x_{22}^{2}, y_{21}\right\rangle+\left\langle A_{21} x_{22}^{1}, y_{21}\right\rangle & \left\langle A_{21} x_{22}^{2}, y_{21}\right\rangle
\end{array}\right] .
$$

We identify the vector space $\Theta$ of all symmetric $2 \times 2$ matrices with the vector space $\mathbb{C}^{3}$ via the isomorphism $\psi: \Theta \longrightarrow \mathbb{C}^{3}$ defined by

$$
\psi(C)=\left[\begin{array}{lll}
c_{11} & c_{12} & c_{22}
\end{array}\right]^{T}
$$

for

$$
C=\left[\begin{array}{ll}
c_{11} & c_{12} \\
c_{12} & c_{22}
\end{array}\right] \in \Theta .
$$

The following theorem gives the structure of basis vectors of $\mathcal{R}_{3}$.

Theorem 3.5. Suppose that $\left\{\mathbf{c}_{l}: 1 \leq l \leq d\right\}$ is a basis for $\mathcal{N}\left(\mathcal{S}_{3}\right)$ and that $C_{l}^{3}=$ $\left[c_{j k l}^{223}\right]_{j, k=1}^{2}=\psi^{-1}\left(\mathbf{c}_{l}\right) \in \Theta$ for $1 \leq l \leq d$. Then we have $d=d_{3}$ and there exist vectors $x_{i 3}^{l} \in \mathcal{D}_{i}^{\prime}, i=1,2, l=1,2, \ldots, d$, such that

$$
\sum_{k=1}^{2} U_{i}\left(\mathbf{c}_{k l}^{23}\right) x_{i 2}^{k}+W_{i}(\boldsymbol{\lambda}) x_{i 3}^{l}=0
$$

where $\mathbf{c}_{k l}^{23}=\left[\begin{array}{ll}c_{1 k l}^{223} & c_{2 k l}^{223}\end{array}\right]^{T}$. The vector

$$
z_{31}=x_{11} \otimes x_{23}^{l}+x_{13}^{l} \otimes x_{21}+\sum_{j, k=1}^{2} c_{j k l}^{223} x_{12}^{j} \otimes x_{22}^{k}
$$

is in $\mathcal{R}_{3} \backslash \mathcal{R}_{2}$, and

$$
\left(\Gamma_{i-1}-\lambda_{i-1} I\right) z_{3 l}=\sum_{k=1}^{2} c_{i k l}^{223} z_{2 k}
$$


for $i=1,2$ and $1 \leq l \leq d$. Moreover, $\left\{z_{1}, z_{21}, z_{22}, z_{31}, z_{32}, \ldots, z_{3 d}\right\}$ is a basis for $\mathcal{R}_{3}$.

Conversely, choose vectors $z_{31}, z_{32}, \ldots, z_{3 d_{2}} \in \mathcal{R}_{3} \backslash \mathcal{R}_{2}$ such that $\left\{z_{1}, z_{21}, z_{22}, z_{31}\right.$, $\left.z_{32}, \ldots, z_{3 d_{3}}\right\}$ is a basis for $\mathcal{R}_{3}$ and such that (3.11) holds for both $i$ and all $l$. Then

$$
C_{l}^{3}=\left[\begin{array}{ll}
c_{11 l}^{223} & c_{12 l}^{223} \\
c_{21 l}^{223} & c_{22 l}^{223}
\end{array}\right]
$$

are symmetric, $\left\{\psi\left(C_{l}^{3}\right): 1 \leq l \leq d_{3}\right\}$ is a basis for $\mathcal{N}\left(\mathcal{S}_{3}\right)$, and there exist vectors $x_{i 3}^{l} \in \mathcal{D}_{i}^{\prime}, i=1,2$, such that (3.9) and (3.10) hold.

Proof. Because $\mathbf{c}_{l} \in \mathcal{N}\left(\mathcal{S}_{3}\right)$ and $C_{l}^{3}=\psi^{-1}\left(\mathbf{c}_{l}\right)$ it follows that

$$
\sum_{j=1}^{2} \sum_{k=1}^{2} c_{j k l}^{223}\left\langle A_{i, j-1} x_{i 2}^{l}, y_{i 1}\right\rangle=0
$$

for $i=1,2$. Thus $\sum_{k=1}^{2} U_{i}\left(\mathbf{c}_{k l}^{23}\right) x_{i 2}^{k} \in\left(\mathcal{N}\left(W_{i}(\boldsymbol{\lambda})^{*}\right)\right)^{\perp}$ and by Lemma 3.3 there exist vectors $x_{i 3} \in \mathcal{D}_{i}^{\prime}$ such that (3.9) hold for $i=1,2$. Next we form the vector $z_{3 l}$ as in (3.10). In the following calculation we assume that $i=0$. For $i=1$ the calculation is similar and we omit it. We have that

$$
\begin{gathered}
\left(\Delta_{0}-\lambda_{0} \Delta_{2}\right) z_{3 l} \\
=\left(A_{11} \otimes W_{2}(\boldsymbol{\lambda})-W_{1}(\boldsymbol{\lambda}) \otimes A_{21}\right)\left(x_{11} \otimes x_{23}^{l}+x_{13}^{l} \otimes x_{21}+\sum_{j, k=1}^{2} c_{j k l}^{223} x_{12}^{j} \otimes x_{22}^{k}\right) \\
=\sum_{j, k=1}^{2} c_{j k l}^{223}\left(A_{1, k-1} x_{12}^{j} \otimes A_{21} x_{21}-A_{11} x_{11} \otimes A_{2, j-1} x_{22}^{k}\right) \\
+\sum_{j, k=1}^{2} c_{j k l}^{223}\left(-A_{11} x_{12}^{j} \otimes A_{2, k-1} x_{21}+A_{1, j-1} x_{11} \otimes A_{21} x_{22}^{k}\right) \\
=\sum_{j, k=1}^{2} c_{j k l}^{223}\left(-A_{11} \otimes A_{2, j-1}+A_{1, j-1} \otimes A_{21}\right)\left(x_{12}^{k}+x_{11} \otimes x_{22}^{k}\right) \\
=\Delta_{2}\left(\sum_{k=1}^{2} c_{1 k l}^{223} z_{2 k}\right) .
\end{gathered}
$$

Conversely, suppose that $z_{3} \in \mathcal{R}_{3} \backslash \mathcal{R}_{2}$. Then there exist a symmetric matrix

$$
C=\left[\begin{array}{ll}
c_{11} & c_{12} \\
c_{12} & c_{22}
\end{array}\right] \in \Theta
$$

and a vector $\mathbf{a}=\left[\begin{array}{ll}a_{0} & a_{1}\end{array}\right]^{T} \in \mathbb{C}^{2}$ such that for $i=0,1$, we have

$$
\left(\Gamma_{i}-\lambda_{i} I\right) z_{3}=\sum_{k=1}^{2} c_{i+1, k} z_{2 k}+a_{i} z_{1} .
$$

We may and will assume that $\mathbf{a}=0$. This is achieved by substituting $z_{3}-a_{0} z_{21}-$ $a_{1} z_{22}$ for $z_{3}$. Thus we have

$$
\left(\Gamma_{i}-\lambda_{i} I\right) z_{3}=\sum_{k=1}^{2} c_{i+1, k} z_{2 k} .
$$


Next it follows from (2.1) that

$$
\sum_{j=0}^{1} A_{i j}^{\dagger}\left(\Gamma_{j}-\lambda_{j} I\right) z_{3}+W_{i}(\boldsymbol{\lambda})^{\dagger} z_{3}=0
$$

and from (3.12) that

$$
\sum_{j=0}^{1} A_{i j}^{\dagger}\left(\sum_{k=1}^{2} c_{j+1, k} z_{2 k}\right)+W_{i}(\boldsymbol{\lambda})^{\dagger} z_{3}=0 .
$$

For $i=1,2$, we choose vectors $v_{i} \in H_{i}$ so that $\left\langle x_{i 1}, v_{i}\right\rangle=1$ and $\left\langle x_{i 2}^{k}, v_{i}\right\rangle=0$ for $k=1,2$. This is possible because $\operatorname{Span}\left\{x_{i 1}\right\} \cap \mathcal{D}_{i}^{\prime}=\{0\}$. For $i=1$ and $i=2$, respectively, we form the scalar product of (3.13) by $y_{11} \otimes v_{2}$ and $v_{1} \otimes y_{21}$, respectively, to get

$$
\sum_{j=0}^{1}\left\langle A_{i j} \sum_{k=1}^{2} c_{j+1, k} x_{i 2}^{k}, y_{i 1}\right\rangle=0 .
$$

Hence, by Lemma 3.3, there exist vectors $x_{i 3} \in \mathcal{D}_{i}^{\prime}$ such that

$$
\sum_{k=1}^{2} U_{i}\left(\mathbf{c}_{k}\right) x_{i 2}^{k}+W_{i}(\boldsymbol{\lambda}) x_{i 3}=0,
$$

where $\mathbf{c}_{k}=\left[\begin{array}{cc}c_{1 k} & c_{2 k}\end{array}\right]^{T}$. Now we form the vector

$$
z_{31}=x_{11} \otimes x_{23}+x_{13} \otimes x_{21}+\sum_{j, k=1}^{2} c_{j k} x_{12}^{j} \otimes x_{22}^{k} .
$$

The same calculation as in the first part of the proof shows that

$$
\left(\Gamma_{i}-\lambda_{i} I\right) z_{31}=\sum_{k=1}^{2} c_{i+1, k} z_{2 k}
$$

for $i=0,1$. It follows that $z_{31}-z_{3} \in \mathcal{R}_{1}$ and so there exists a number $\delta \in \mathbb{C}$ such that $z_{3}=z_{31}+\delta z_{1}$. Without loss, we can use the vector $x_{13}+\delta x_{11}$ in place of $x_{13}$. Then it follows that

$$
z_{3}=x_{11} \otimes x_{23}+x_{13} \otimes x_{21}+\sum_{j, k=1}^{2} c_{j k} x_{12}^{j} \otimes x_{22}^{k} .
$$

The final step is to verify that $\psi(T) \in \mathcal{N}\left(\mathcal{S}_{3}\right)$, which follows immediately from the equalities (3.14).

Note that Theorem 3.1 implies the existence of vectors $z_{3 l} \in \mathcal{R}_{3} \backslash \mathcal{R}_{2}\left(1 \leq l \leq d_{3}\right)$ such that the following hold:

- $\left\{z_{1}, z_{21}, z_{22}, z_{31}, z_{32}, \ldots, z_{3 d_{3}}\right\}$ is a basis for $\mathcal{R}_{3}$,

- equality (3.11) holds for both $i$ and all $l$,

- $\left\{C_{l}^{3}: 1 \leq l \leq d_{3}\right\}$ are linearly independent.

Since $\Gamma_{0}$ and $\Gamma_{1}$ commute it follows that $c_{12 l}^{223}=c_{21 l}^{223}\left(1 \leq l \leq d_{3}\right)$ and therefore the matrices $C_{l}^{3}$ are symmetric. 


\section{A basis for root subspace $\mathcal{R}_{m}, m \geq 3$, At a Derogatory eigenvalue}

Our goal in this section is to prove, by induction on $m$, the following theorem which is our main result.

Theorem 4.1. Suppose that $\left\{\mathbf{c}_{1 f}^{m}, f=1,2, \ldots, d\right\}$ is a basis for the kernel of $\mathcal{S}_{m}$. Then $d=d_{m}$ and there exist vectors $x_{i m}^{f} \in \mathcal{D}_{i}^{\prime}$ and matrices $C_{f}^{m}$ such that conditions ( $i$ ) to (iii) below hold when $m-1$ is replaced by $m$. In particular, the union $\mathcal{B}_{m}=\mathcal{B}_{m-1} \cup\left\{z_{m 1}, z_{m 2}, \ldots, z_{m d}\right\}$, where

(4.1) $z_{m f}=x_{1 m}^{f} \otimes x_{21}+x_{11} \otimes x_{2 m}^{f}+\sum_{k=2}^{\left[\frac{m}{2}\right]} \sum_{l=k}^{m+1-k} \sum_{g=1}^{d_{k}} \sum_{h=1}^{d_{l}} c_{g h f}^{k l m}\left(x_{1 k}^{g} \otimes x_{2 l}^{h}+x_{1 l}^{h} \otimes x_{2 k}^{g}\right)$

for $f=1,2, \ldots, d$, is a basis for the root subspace $\mathcal{R}_{m}$.

Here $\left[\frac{m}{2}\right]$ denotes the integer part of $\frac{m}{2}$.

We will prove Theorem 4.1 at the end of the section. Let us first introduce the inductive assumptions. For $m=1,2,3, \ldots$ we suppose that we have vectors $x_{i 1} \in$ $\mathcal{D}\left(A_{i 0}\right), x_{i l}^{h} \in \mathcal{D}_{i}^{\prime}, i=1,2, l=2,3, \ldots, m-1, h=1,2, \ldots, d_{l}$, and symmetric (structure) matrices (see (3.4))

$$
C_{h}^{l}=\left[\begin{array}{ccccc}
C_{h}^{22 l} & C_{h}^{23 l} & \cdots & C_{h}^{2, l-2, l-1} & C_{h}^{2, l-1, l} \\
\left(C_{h}^{23 l}\right)^{T} & C_{h}^{33 l} & \cdots & C_{h}^{3, l-2, l} & 0 \\
\vdots & \vdots & & \vdots & \vdots \\
\left(C_{h}^{2, l-2, l}\right)^{T} & \left(C_{h}^{3, l-2, l}\right)^{T} & \cdots & 0 & 0 \\
\left(C_{h}^{2, l-1, l}\right)^{T} & 0 & \cdots & 0 & 0
\end{array}\right]
$$

such that

(i) the matrices $C_{h}^{2, l-1, l}, h=1,2, \ldots, d_{l}$, are linearly independent for $l=$ $3,4, \ldots, m-1$, the column cross-sections (see (3.3) ) $\mathcal{C}_{h}^{j l}$ are equal to $C_{h}^{2 j l}$, and the entries of matrices $C_{h}^{l}$ satisfy the relations

$$
\sum_{k=l_{1}+l_{2}-1}^{l-l_{3}+1} \sum_{g=1}^{d_{k}} c_{h_{1} h_{2} g}^{l_{1} l_{2} k} c_{h_{3} g h}^{l_{3} k l}=\sum_{k=l_{2}+l_{3}-1}^{l-l_{1}+1} \sum_{g=1}^{d_{k}} c_{h_{1} h_{3} g}^{l_{1} l_{3} k} c_{h_{2} g h}^{l_{2} k l}
$$

for $l=4,5, \ldots, m-1, \mathbf{l} \in \Phi_{l}$ and $\mathbf{h} \in \chi_{\mathbf{l}}$,

(ii) the relations (2.2), 3.6) and

$$
\sum_{k=2}^{l-1} \sum_{g=1}^{d_{k}} U_{i}\left(\mathbf{c}_{g h}^{k l}\right) x_{i k}^{g}+W_{i}(\boldsymbol{\lambda}) x_{i l}^{h}=0
$$

for $i=1,2, l=3,4, \ldots, m-1$ and $h=1,2, \ldots, d_{l}$ hold (here $\mathbf{c}_{g h}^{k l}=$ $\left[\begin{array}{ll}c_{1 g h}^{2 k l} & c_{2 g h}^{2 k l}\end{array}\right]^{T}$ ),

(iii) the vectors $z_{1}, z_{21}, z_{22}$, of (2.3) and (3.7) together with vectors $z_{31}, z_{32}$, $\ldots, z_{3 d_{3}}, \ldots, z_{m-1,1}, z_{m-1,2}, \ldots, z_{m-1, d_{m-1}}$, that are defined by

$z_{l h}=x_{1 l}^{h} \otimes x_{21}+x_{11} \otimes x_{2 l}^{h}+\sum_{k=2}^{\left[\frac{l}{2}\right]} \sum_{r=k}^{l+1-k} \sum_{g=1}^{d_{k}} \sum_{e=1}^{d_{r}} c_{g e h}^{k r l}\left(x_{1 k}^{g} \otimes x_{2 r}^{e}+x_{1 r}^{e} \otimes x_{2 k}^{g}\right)$ 
for $l=3,4, \ldots, m-1$ and $h=1,2, \ldots, d_{l}$, form a filtered basis for the root subspace $\mathcal{R}_{m-1}$. We also write

$$
\mathcal{B}_{m-1}=\left\{z_{1}, z_{21}, z_{22}, \ldots, z_{m-1,1}, z_{m-1,2}, \ldots, z_{m-1, d_{m-1}}\right\} .
$$

By Theorem [3.5 it follows that there exist vectors $x_{i 1}, x_{i 2}^{h}, x_{i 3}^{h}$ and matrices $C_{2}^{h}$ such that conditions $(i)$ to (iii) are satisfied for $m-1=3$. Now we assume that the above conditions are satisfied for $m-1$ (with $3 \leq m-1 \leq M-1$ ) and we prove that we can find vectors $x_{i m}^{f} \in \mathcal{D}_{i}^{\prime}, i=1,2, f=1,2, \ldots, d_{m}$, and matrices $C_{f}^{m}, f=1,2, \ldots, d_{m}$, such that (i) to (iii) hold with $m-1$ replaced by $m$.

Our next step is to introduce matrices $\mathcal{S}_{m}$. We do this in two stages. First we use matrices $C_{g}^{k}, k<m$, and second, certain orthogonality relations.

For the purpose of calculation we write $T^{m}=\left[T^{k l m}\right]_{k, l=2}^{m-1}$ for an unknown symmetric matrix in the form (4.2). The entries of the $d_{k} \times d_{l}$ matrix $T^{k l m}$ are denoted by $t_{g h}^{k l m}$. They must satisfy the conditions

$$
\sum_{k=l_{1}+l_{2}-1}^{m-l_{3}+1} \sum_{g=1}^{d_{k}} c_{h_{1} h_{2} g}^{l_{1} l_{2} k} t_{h_{3} g}^{l_{3} k m}-\sum_{k=l_{1}+l_{3}-1}^{m-l_{2}+1} \sum_{g=1}^{d_{k}} c_{h_{1} h_{3} g}^{l_{1} l_{3} k} t_{h_{2} g}^{l_{2} k m}=0
$$

for $\mathbf{l} \in \Phi_{m}$ and $\mathbf{h} \in \chi_{\mathbf{l}}$. We write the $2 \times d_{m-1}$ matrix $T^{1, m-1, m}$ also as a column (4.6)

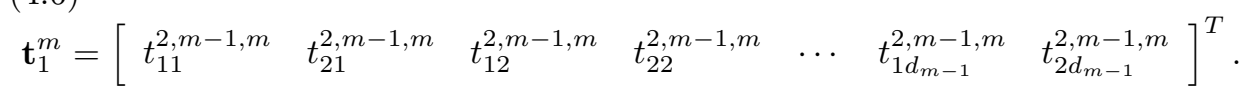

We denote the sum $\sum_{i=1}^{l} d_{i}$ by $\widehat{d}_{l}$. For every column of $T^{m}$ we define a column vector $\mathbf{t}_{k g}^{m}, k=2,3, \ldots, m-1, g=1,2, \ldots, d_{k}$, of size $\nu=\min \left\{\widehat{d}_{k-1}+g, \widehat{d}_{m-k}\right\}$ by taking the first $\nu$ entries in the $\left(\widehat{d}_{k-1}+g\right)$-th column of $T^{m}$. Note that $\mathbf{t}_{k g}^{m}$ are defined so that they consist of all the entries above and including the main diagonal of the matrix $T^{m}$ omitting those labelled 0 in (4.2). We define a column vector $\mathbf{t}_{2}^{m}$ by

$$
\begin{array}{rllllll}
\mathbf{t}_{2}^{m}=\left[\begin{array}{llllll}
\left(\mathbf{t}_{11}^{m}\right)^{T} & \left(\mathbf{t}_{12}^{m}\right)^{T} & \left(\mathbf{t}_{21}^{m}\right)^{T} & \cdots & \left(\mathbf{t}_{2 d_{2}}^{m}\right)^{T} & \cdots \\
& & & \left(\mathbf{t}_{m-2,1}^{m}\right)^{T} & \cdots & \left(\mathbf{t}_{m-2, d_{m-2}}^{m}\right)^{T}
\end{array}\right]^{T} .
\end{array}
$$

We split the entries of the matrix $T^{m}$ into two column vectors $\mathbf{t}_{1}^{m}$ and $\mathbf{t}_{2}^{m}$. The mapping $\psi_{m}: T^{m} \mapsto\left(\mathbf{t}_{1}^{m}, \mathbf{t}_{2}^{m}\right)$, defined on all symmetric matrices of the form (4.2), is a generalization of the transformation $\psi$ defined by (3.8). It is bijective and therefore it has an inverse, which maps two vectors $\mathbf{t}_{1}^{m}$ and $\mathbf{t}_{2}^{m}$ into a matrix $T^{m}$ of the form (4.2). We use this inverse mapping in Lemma 4.3 We write the system of equations (4.5) in matrix form as

$$
S_{m}^{21} \mathbf{t}_{1}^{m}+S_{m}^{22} \mathbf{t}_{2}^{m}=0
$$

where the entries of the matrices $S_{m}^{21}$ and $S_{m}^{22}$ are determined by the system 4.5. These entries are given because we assumed that the matrices $C_{g}^{k}$ were known for $k \leq m-1$.

We also want the entries of the matrices $T^{2 k m}$ to satisfy the scalar relations

$$
\sum_{k=2}^{m-1} \sum_{g=1}^{d_{k}}\left\langle\left(t_{1 g}^{2 k m} A_{i 0}+t_{2 g}^{2 k m} A_{i 1}\right) x_{i k}^{g}, y_{i 1}\right\rangle=0
$$


for both $i$. These can be written equivalently in matrix form

$$
S_{m}^{11} \mathbf{t}_{1}^{m}+S_{m}^{12} \mathbf{t}_{2}^{m}=0 \text {. }
$$

Again the entries of the matrices $S_{m}^{11}$ and $S_{m}^{12}$ are determined by the equations (4.9).

Now we are prepared to define the matrices $\mathcal{S}_{m}$. First we choose a matrix $B_{m}$ so that its columns form a basis for the kernel of

$$
\left[\left(S_{m}^{12}\right)^{*}\left(S_{m}^{22}\right)^{*}\right] \text {. }
$$

Then we define a matrix

$$
\mathcal{S}_{m}=B_{m}^{*}\left[\begin{array}{c}
S_{m}^{11} \\
S_{m}^{21}
\end{array}\right]
$$

so equations (4.8) and (4.10) yield $\mathcal{S}_{m} \mathbf{t}_{1}^{m}=0$.

We now choose vectors $z_{m f} \in \mathcal{D}, f=1,2, \ldots, d_{m}$, so that $\mathcal{B}_{m-1} \cup\left\{z_{m 1}, z_{m 2}, \ldots\right.$, $\left.z_{m d_{m}}\right\}$, is a basis for the space $\mathcal{R}_{m}$. By Theorem 3.1 there exist matrices $C_{f}^{m}$ for $f=1,2, \ldots, d_{m}$ such that $(i)$ holds and that we have

$$
\left(\Gamma_{i-1}-\lambda_{i-1} I\right) z_{m f}=\sum_{k=2}^{m-1} \sum_{g=1}^{d_{k}} c_{i g f}^{2 k m} z_{k g}
$$

for $i=1,2$ and $f=1,2, \ldots, d_{m}$. Next we prove three auxiliary results.

Lemma 4.2. In the above setting it follows that $\operatorname{dim} \mathcal{N}\left(\mathcal{S}_{m}\right) \geq d_{m}$.

Proof. Let $\psi_{m}\left(C_{f}^{m}\right)=\left(\mathbf{c}_{1 f}^{m}, \mathbf{c}_{2 f}^{m}\right)$. By Theorem 3.1(e) and the definition of $\psi_{m}$ it follows that $S_{m}^{21} \mathbf{c}_{1 f}^{m}+S_{m}^{22} \mathbf{c}_{2 f}^{m}=0$. Relation (2.1) implies

$$
\sum_{j=0}^{1} A_{i j}^{\dagger}\left(\Gamma_{j}-\lambda_{j} I\right) z_{m f}+W_{i}(\boldsymbol{\lambda})^{\dagger} z_{m f}=0
$$

for both $i$. By relations (4.11) we get that

$$
\sum_{k=2}^{m-1} \sum_{g=1}^{d_{k}} U_{i}\left(\mathbf{c}_{g f}^{k m}\right)^{\dagger} z_{k g}+W_{i}(\boldsymbol{\lambda})^{\dagger} z_{m f}=0 .
$$

Here $\mathbf{c}_{g f}^{k m}=\left[\begin{array}{ll}c_{1 g f}^{2 k m} & c_{2 g f}^{2 k m}\end{array}\right]^{T}$. Because we assume that $x_{i k}^{g} \in \mathcal{D}_{i}^{\prime}$ and $\mathcal{D}_{i}^{\prime} \cap$ $\operatorname{Span}\left\{x_{i 1}\right\}=\{0\}$ it follows that for each $i$ there exists a vector $v_{i} \in H_{i}$ such that $\left\langle x_{i 1}, v_{i}\right\rangle=1$ and $\left\langle x_{i k}^{g}, v_{i}\right\rangle=0$ for $k=2,3, \ldots, m-1$ and $g=1,2, \ldots, d_{k}$. Then the scalar products of (4.12) by $y_{11} \otimes v_{2}$ and $v_{1} \otimes y_{21}$, for $i=1$ and $i=2$ respectively, are equal to 0 . It follows, using the structure of the vectors $z_{k g}$ in condition (iii), that

$$
\sum_{k=2}^{m-1} \sum_{g=1}^{d_{k}}\left\langle U_{i}\left(\mathbf{c}_{g f}^{k m}\right) x_{i k}^{g}, y_{i 1}\right\rangle=0
$$

for all $f$, and so

$$
\sum_{j=1}^{2} \sum_{k=2}^{m-1} \sum_{g=1}^{d_{k}} c_{j g f}^{2 k m}\left\langle A_{i, j-1} x_{i k}^{g}, y_{i 1}\right\rangle=0 .
$$

Therefore $\mathbf{c}_{1 f}^{m}$ and $\mathbf{c}_{2 f}^{m}$ solve equation (4.10) and $\mathbf{c}_{1 f}^{m}, f=1,2, \ldots, d_{m}$, are elements of the kernel of the matrix $\mathcal{S}_{m}$. Since these vectors are linearly independent, we have $d_{m} \leq \operatorname{dim} \mathcal{N}\left(\mathcal{S}_{m}\right)$. 
Lemma 4.3. Suppose that $\mathbf{c}_{11}^{m}$ is an element of the kernel $\mathcal{N}\left(\mathcal{S}_{m}\right)$. Then there exists a vector $\mathbf{c}_{21}^{m}$ such that (4.8) and (4.10) hold. Furthermore, there exist vectors $x_{i m}^{1} \in \mathcal{D}_{i}^{\prime}, i=1,2$, such that

$$
\sum_{k=2}^{m-1} \sum_{g=1}^{d_{k}} U_{i}\left(\mathbf{c}_{g 1}^{k m}\right) x_{i k}^{g}+W_{i}(\boldsymbol{\lambda}) x_{i m}^{1}=0 .
$$

Here $\mathbf{c}_{g 1}^{k m}$ are determined by the first block column of $C_{1}^{m}=\psi_{m}^{-1}\left(\mathbf{c}_{1}^{m}, \mathbf{c}_{2}^{m}\right)$.

Proof. From the structure of the matrix $\mathcal{S}_{m}$ it follows that for an element $\mathbf{c}_{11}^{m} \in$ $\mathcal{N}\left(\mathcal{S}_{m}\right)$ there exists a vector $\mathbf{c}_{21}^{m}$ such that relations (4.8) and (4.10) hold. Using the inverse of the isomorphism $\psi_{m}^{-1}$ we associate with the pair of vectors $\mathbf{c}_{11}^{m}$ and $\mathbf{c}_{21}^{m}$ a matrix $C_{1}^{m}$. The relations (4.10) can be written equivalently in the form (4.13). Then it follows for both $i$ that

$$
\sum_{k=2}^{m-1} \sum_{g=1}^{d_{k}} U_{i}\left(\mathbf{c}_{g 1}^{k m}\right) x_{i k}^{g} \in\left(\mathcal{N}\left(W_{i}(\boldsymbol{\lambda})^{*}\right)\right)^{\perp}=\mathcal{R}\left(W_{i}(\boldsymbol{\lambda})\right) .
$$

The latter equality holds since $W_{i}(\boldsymbol{\lambda})$ is a Fredholm operator. By Lemma 3.3 there exists a vector $x_{i m}^{1} \in \mathcal{D}_{i}^{\prime}$ such that (4.14) holds.

Lemma 4.4. Suppose that we have the same setting as in Lemma[4.3. We construct a vector

$$
z_{m 1}=x_{1 m}^{1} \otimes x_{21}+x_{11} \otimes x_{2 m}^{1}+\sum_{k=1}^{\left[\frac{m}{2}\right]} \sum_{l=k}^{m+1-k} \sum_{g=1}^{d_{k}} \sum_{h=1}^{d_{l}} c_{g h 1}^{k l m}\left(x_{1 k}^{g} \otimes x_{2 l}^{h}+x_{1 l}^{h} \otimes x_{2 k}^{g}\right) .
$$

Then it follows that

$$
\left(\Gamma_{j-1}-\lambda_{j-1} I\right) z_{m 1}=\sum_{k=2}^{m-1} \sum_{g=1}^{d_{k}} c_{j g 1}^{2 k m} z_{k g}
$$

for $j=1,2$.

Proof. We use a direct calculation to show (4.15). We consider only the case $j=1$. The case $j=2$ is proved in the same way. By the basic properties of operator determinants we have

$$
\begin{aligned}
& \left(\Delta_{0}-\lambda_{0} \Delta_{2}\right) z_{m 1}-\sum_{k=2}^{m-1} \sum_{g=1}^{d_{k}} c_{1 g 1}^{2 k m} \Delta_{2} z_{k g} \\
& =\left|\begin{array}{ll}
-W_{1}(\boldsymbol{\lambda})^{\dagger} & A_{11}^{\dagger} \\
-W_{2}(\boldsymbol{\lambda})^{\dagger} & A_{21}^{\dagger}
\end{array}\right|\left(x_{1 m}^{1} \otimes x_{21}+x_{11} \otimes x_{2 m}^{1}\right) \\
& -\sum_{k=2}^{m-1} \sum_{g=1}^{d_{k}}\left|\begin{array}{ll}
U_{1}\left(\mathbf{c}_{g 1}^{k m}\right)^{\dagger} & A_{11}^{\dagger} \\
U_{2}\left(\mathbf{c}_{g 1}^{k m}\right)^{\dagger} & A_{21}^{\dagger}
\end{array}\right|\left(x_{1 k}^{g} \otimes x_{21}+x_{11} \otimes x_{2 k}^{g}\right) \\
& +\left|\begin{array}{ll}
-W_{1}(\boldsymbol{\lambda})^{\dagger} & A_{11}^{\dagger} \\
-W_{2}(\boldsymbol{\lambda})^{\dagger} & A_{21}^{\dagger}
\end{array}\right| \sum_{k=2}^{\left[\frac{m}{2}\right]} \sum_{l=k}^{m+1-k} \sum_{g=1}^{d_{k}} \sum_{h=1}^{d_{l}} c_{g h 1}^{k l m}\left(x_{1 k}^{g} \otimes x_{2 l}^{h}+x_{1 l}^{h} \otimes x_{2 k}^{g}\right) \\
& -\sum_{k=3}^{m-1} \sum_{g=1}^{d_{k}} c_{1 g 1}^{2 k m} \sum_{l=2}^{\left[\frac{k}{2}\right]} \sum_{r=l}^{k+1-l} \sum_{h=1}^{d_{l}} \sum_{e=1}^{d_{r}} c_{e h g}^{r l k} \Delta_{2}\left(x_{1 r}^{e} \otimes x_{2 l}^{h}+x_{1 l}^{h} \otimes x_{2 r}^{e}\right) .
\end{aligned}
$$


Relation (4.14) implies that the sum of (4.17) and (4.18) is equal to 0. Since $c_{e h g}^{r l k}=c_{\text {heg }}^{l r k}$ it follows that (4.20) is equal to

$$
\begin{gathered}
-\sum_{k=3}^{m-1} \sum_{l=1}^{k-1} \sum_{r=l}^{k+1-l} \sum_{g=1}^{d_{k}} \sum_{h=1}^{d_{l}} \sum_{e=1}^{d_{r}} c_{1 g 1}^{2 k m} c_{e h g}^{r l k} \Delta_{2} x_{1 r}^{e} \otimes x_{2 l}^{h} \\
=-\sum_{r=2}^{m-2} \sum_{l=2}^{r-1} \sum_{h=1}^{d_{l}} \sum_{e=1}^{d_{r}}\left[\sum_{k=l+r-1}^{m-1} \sum_{g=1}^{d_{k}} c_{1 g 1}^{2 k m} c_{e h g}^{r l k}\right] \Delta_{2} x_{1 r}^{e} \otimes x_{2 l}^{h} .
\end{gathered}
$$

We use relations (3.5) and the definition of $\Delta_{2}$, and we rearrange the order of summation to show that (4.21) is equal to

$$
-\sum_{k=2}^{\left[\frac{m}{2}\right]} \sum_{l=k}^{m+1-k} \sum_{g=1}^{d_{k}} \sum_{h=1}^{d_{l}} \sum_{r=k}^{l-1} \sum_{e=1}^{d_{r}} c_{g h 1}^{k l m}\left|\begin{array}{cc}
U_{1}\left(\mathbf{c}_{e g}^{r k}\right)^{\dagger} & A_{11}^{\dagger} \\
U_{2}\left(\mathbf{c}_{e g}^{r k}\right)^{\dagger} & A_{21}^{\dagger}
\end{array}\right|\left(x_{1 r}^{e} \otimes x_{2 l}^{h}+x_{1 l}^{h} \otimes x_{2 r}^{e}\right) .
$$

If we use (4.4) and (4.14) we see that the sum of (4.19) and 4.22) is 0 . Therefore (4.16) is equal to 0 , and (4.15) follows.

Now we are ready to prove our main result.

Proof of Theorem 4.1. Suppose that we are given a basis $\left\{\mathbf{c}_{1 f}^{m}, f=1,2, \ldots, d\right\}$ for $\mathcal{N}\left(\mathcal{S}_{m}\right)$. Then by Lemma 4.3 it follows that we can find vectors $x_{i m}^{f} \in \mathcal{D}_{i}^{\prime}$ and matrices $C_{f}^{m}$ of the form (4.2), where $C_{f}^{m}=\psi_{m}^{-1}\left(\mathbf{c}_{1 f}^{m}, \mathbf{c}_{2 f}^{m}\right)$ for some $\mathbf{c}_{2 f}^{m}$, such that (i) and (ii) hold when $m-1$ is replaced by $m$. We apply Lemma 4.4 to show that for $f=1,2, \ldots, d$ the vectors $z_{m f}$ are in $\mathcal{R}_{m}$ but not in $\mathcal{R}_{m-1}$. They are linearly independent because $\mathbf{c}_{1 f}^{m}, f=1,2, \ldots, d$, are linearly independent. It follows that $d \leq d_{m}$ and, because $d \geq d_{m}$ by Lemma 4.2, we have $d=d_{m}$. Thus (iii) holds also.

\section{The Sturm-Liouville Case}

Faierman [8] considers a two-parameter eigenvalue problem involving a class of coupled Sturm-Liouville boundary value problems

$$
\begin{aligned}
& \frac{d}{d t_{i}}\left(p_{i}\left(t_{i}\right) \frac{d y_{i}}{d t_{i}}\right)+\left((-1)^{i+1} \mu_{1} a_{i 1}\left(t_{i}\right)+(-1)^{i} \mu_{2} a_{i 2}\left(t_{i}\right)-q_{i}\left(t_{i}\right)\right) y_{i}=0, i=1,2, \\
& (5.2) \quad y_{i}(0) \cos \alpha_{i}-p_{i}(0) \frac{d y_{i}}{d t_{i}}(0) \sin \alpha_{i}=0,0 \leq \alpha_{i}<\pi, i=1,2,
\end{aligned}
$$

and

$$
y_{i}(1) \cos \beta_{i}-p_{i}(1) \frac{d y_{i}}{d t_{i}}(1) \sin \beta_{i}=0,0<\beta_{i} \leq \pi, i=1,2,
$$

where $\boldsymbol{\mu}=\left(\mu_{1}, \mu_{2}\right)$ are parameters, $\mathcal{I}=[0,1]$ and $t_{i} \in \mathcal{I}$. As in [8, pp. 2 and 10] we assume that

(i) for $i=1,2$ the functions $p_{i}, q_{i}, a_{i j}, j=1,2$, are real-valued, $p_{i}, a_{i j}$ are Lipschitz continuous, $p_{i}$ is positive and $q_{i}$ is essentially bounded,

(ii) the function $\omega\left(t_{1}, t_{2}\right)=a_{11}\left(t_{1}\right) a_{22}\left(t_{2}\right)-a_{12}\left(t_{1}\right) a_{21}\left(t_{2}\right)$ on $\mathcal{I}^{2}$ is not identically 0 ,

(iii) $a_{i 1}\left(t_{i}\right)>0$ for $t_{i} \in \mathcal{I}$ and $i=1,2$. 
Weaker assumptions would be possible (cf. $[4,5,26]$ ) but we adhere to Faierman's assumptions for the purpose of comparison.

Two-parameter problems which satisfy condition (iii) are called elliptic. In the literature also a formally stronger condition

(iii') each $a_{i j}\left(t_{i}\right)$ is positively bounded below on $\mathcal{I}$

is considered (e.g. [3]). Two-parameter problems which satisfy condition (iii') are called uniformly elliptic. We show in Lemma 5.1 that we can transform an elliptic problem to a uniformly elliptic one by a linear substitution in parameters.

To introduce the operators $A_{i j}$ we take $H_{i}=L_{2}(\mathcal{I})$. We have

$$
A_{i j} y_{i}\left(t_{i}\right)=a_{i j}\left(t_{i}\right) y_{i}\left(t_{i}\right), \quad i, j=1,2 \text {, }
$$

for $y_{i} \in H_{i}$ and

$$
A_{i 0} y_{i}\left(t_{i}\right)=\frac{d}{d t_{i}}\left(p_{i}\left(t_{i}\right) \frac{d y_{i}}{d t_{i}}\right)-q_{i}\left(t_{i}\right) y_{i}, \quad i=1,2,
$$

where the domain $\mathcal{D}\left(A_{i 0}\right)$ consists of all functions $y_{i} \in H_{i}$ such that $y_{i}$ and $\frac{d y_{i}}{d t_{i}}$ are absolutely continuous, $\frac{d^{2} y_{i}}{d t_{i}{ }^{2}} \in H_{i}$ and the boundary conditions (5.2) and (5.3) hold.

Lemma 5.1. Assume that the problem (5.1)-(5.3) is such that (i)-(iii) hold. Then there exists an invertible linear substitution of parameters $\boldsymbol{\mu}$ such that the problem is uniformly elliptic after the substitution.

Proof. The numerical range

$$
\kappa_{i}=\left\{\left(c_{i 1}, c_{i 2}\right) \in \mathbb{R}^{2}: \text { for } j=1,2 c_{i j}=\left(A_{i j} x_{i}, x_{i}\right) \text { for some } x_{i} \in H_{i},\left\|x_{i}\right\|=1\right\}
$$

is a bounded convex set for $i=1,2$. By conditions (i) and (iii) it follows that for some $\epsilon>0$ we have $c_{11}>\epsilon$ for all elements of $\kappa_{1}$ and $c_{21}<-\epsilon$ for all elements of $\kappa_{2}$. Then there exist two lines through the origin in $\mathbb{R}^{2}$ that separate $\kappa_{1}$ and $\kappa_{2}$ and we can find an invertible linear map $\tau$ on $\mathbb{R}^{2}$ that maps $\kappa_{1}$ and $\kappa_{2}$ into the first and the third quadrant, respectively. If we apply $\tau$ to the parameters $\boldsymbol{\mu}$ it follows that the transformed problem is uniformly elliptic.

We hereafter assume, without loss of generality, that the parameters $\boldsymbol{\mu}$ are chosen

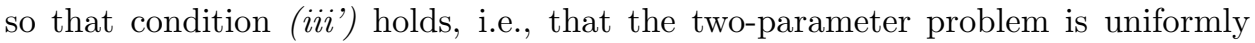
elliptic. In order to apply the results of the previous sections we need to verify Assumptions I-III. By [8, Thm. 2.4] it follows that $\Delta_{2}$ is a selfadjoint operator with dense domain $\mathcal{D}$ of those functions in the Sobolev space $W_{2}^{2}\left(\mathcal{I}^{2}\right)$ that satisfy the boundary condition on $\mathcal{I}^{2}$ associated with (5.2) and (5.3). For details see 8 pp. 10-30]. Next we have:

Lemma 5.2. $\mathcal{N}\left(\Delta_{0}\right) \cap \mathcal{N}\left(\Delta_{2}\right)=\{0\}$.

Proof. Assume that $\Delta_{0} u=\Delta_{2} u=0$ for some $u \in \mathcal{D}$. By (ii) it follows that $\omega$ is nonzero on some open set $\Omega \subset \mathcal{I}^{2}$. Hence $\left.u\right|_{\Omega} \equiv 0$. By the unique continuation property for $\Delta_{2}$ [8] Prop. 3.1] it follows that $u \equiv 0$ on $\mathcal{I}^{2}$.

Now it follows from [5] Prop. 2.1] that Assumption I holds for an $\alpha \in \mathbb{R}$. As in $\S 2$ we shall hereafter assume, without loss of generality, that $\alpha=0$, i.e., that $\Delta_{2}$ has a bounded inverse. This is achieved by a shift in parameters. Assumption II is a consequence of the following lemma. This is given in a special case in [5, §9.3]. Since the argument there appears to be incomplete we provide a more detailed proof here. 
Lemma 5.3. The system

$$
\left[\begin{array}{ll}
A_{10}^{\dagger} & A_{11}^{\dagger} \\
A_{20}^{\dagger} & A_{21}^{\dagger}
\end{array}\right]\left[\begin{array}{l}
u_{1} \\
u_{2}
\end{array}\right]=\left[\begin{array}{l}
A_{12}^{\dagger} z \\
A_{22}^{\dagger} z
\end{array}\right]
$$

has a solution $u=\left(u_{1}, u_{2}\right) \in \mathcal{D}^{2}$ for all $z \in \mathcal{D}$.

Proof. By eliminating $u_{2}$ in (5.6) we have

$$
-\Delta_{2} u_{1}=\Delta_{0} z .
$$

By [8, Thm. 2.2] there exists $u_{1} \in \mathcal{D}$ which solves (5.7). Similarly, the elimination of $u_{2}$ leads to $-\Delta_{2} u_{2}=\Delta_{1} z$, which again is soluble for $u_{2} \in \mathcal{D}$ by $[8$, Thm. 2.2]. Thus it remains to show that $u_{1}$ and $u_{2}$ satisfy (5.6).

We shall do this first under the assumption that each $A_{i 0}$ is positive definite. For $N \in \mathbb{N}$ we define bounded selfadjoint operators $A_{i 0}^{(N)}$ sharing the same eigenvectors and the lowest $N$ eigenvalues with $A_{i 0}$. The remaining eigenvalues of $A_{i 0}^{(N)}$ are defined as 1 . The definition of $A_{i 0}^{(N)}$ is completed by linearity and continuity to $H_{i}$. We define $\Delta_{j}^{(N)}, j=1,2$, analogously to $\Delta_{j}$ and we note that $A_{i 0}^{(N)}$ and $\Delta_{j}^{(N)}$ are uniformly bounded with inverses bounded uniformly in $N$ for $i, j=1,2$.

Now consider the system of equations

$$
A_{i 0}^{(N)}{ }^{\dagger} z_{0}=A_{i 1}^{\dagger} z_{1}+A_{i 2}^{\dagger} z_{2}, \quad i=1,2,
$$

where $z_{2}=\left(\Delta_{1}^{(N)}\right)^{-1} w$ for a fixed $w \in H$. Proceeding as in the proof of [2, Thms. 4.2 and 6.1$]$ we obtain

$$
z_{0}=-\left(\Delta_{2}^{(N)}\right)^{-1} \Delta_{0}\left(\Delta_{1}^{(N)}\right)^{-1} w \text { and } z_{1}=-\left(\Delta_{2}^{(N)}\right)^{-1} w
$$

so (5.8) yields

$$
A_{i 0}^{(N)}{ }^{\dagger} x_{N}=-A_{i 1}^{\dagger}\left(\Delta_{2}^{(N)}\right)^{-1} w-A_{i 2}^{\dagger}\left(\Delta_{1}^{(N)}\right)^{-1} w, \quad i=1,2,
$$

where $x_{N}=\left(\Delta_{2}^{(N)}\right)^{-1} \Delta_{0}\left(\Delta_{1}^{(N)}\right)^{-1} w$. Now [2, Lemma 4.1] shows that the righthand side in (5.9) has limit $-A_{i 1}^{\dagger} \Delta_{2}^{-1} w-A_{i 2}^{\dagger} \Delta_{1}^{-1} w=y_{i}$, say, as $N \rightarrow \infty$. Thus $A_{i 0}^{(N)^{\dagger}} x_{N}$ converges to $y_{i}$. Since the inverses of $A_{i 0}^{(N)}$ are bounded uniformly in $N$ it follows that $x_{N}-\left(\left(A_{i 0}^{(N)}\right)^{-1}\right)^{\dagger} y_{i} \rightarrow 0$ as $N \rightarrow \infty$. Similarly, since the inverses of $\left(\Delta_{2}^{(N)}\right)^{-1}$ are bounded uniformly in $N$ it follows that $x_{N} \rightarrow \Delta_{2}^{-1} \Delta_{0} \Delta_{1}^{-1} w$ as $N \rightarrow \infty$ (cf. [2, Lemma 4.1]). Thus

$$
\Delta_{2}^{-1} \Delta_{0} \Delta_{1}^{-1} w=\left(A_{i 0}^{-1}\right)^{\dagger} y_{i}
$$

whence

$$
A_{i 0}^{\dagger} \Delta_{2}^{-1} \Delta_{0} \Delta_{1}^{-1} w=-A_{i 1}^{\dagger} \Delta_{2}^{-1} w-A_{i 2}^{\dagger} \Delta_{1}^{-1} w .
$$

Now put $w=\Delta_{1} z$ to obtain (5.6) for $u_{1}=-\Delta_{2}^{-1} \Delta_{0} z$ and $u_{2}=-\Delta_{2}^{-1} \Delta_{1} z$. To remove the restriction that $A_{i 0}$ are uniformly positive we use the technique of [6] Thms. 3.1 and 3.2]. 
In the rest of this section we discuss the eigenvalues and associated root subspaces of (5.1)-(5.3). If there exist nonzero functions $y_{i} \in \mathcal{D}\left(A_{i 0}\right), i=1,2$, which solve (5.1)-(5.3) for a pair $\boldsymbol{\mu}=\left(\mu_{1}, \mu_{2}\right) \in \mathbb{C}^{2}$, then $\boldsymbol{\mu}$ is called an eigenvalue for the problem (5.1)-(5.3). The function $w\left(t_{1}, t_{2}\right)=y_{1}\left(t_{1}\right) y_{2}\left(t_{2}\right)$ is such that $\left(\Delta_{i}-\mu_{i} \Delta_{0}\right) w=0$ for $i=1,2$. Because we assume that $\Delta_{2}$ has a bounded inverse, and in particular, is one-to-one, we have that $\mu_{2} \neq 0$. Then it follows for each eigenvalue $\boldsymbol{\mu}$ of (5.1)-(5.3) that $\boldsymbol{\lambda}=\left(\lambda_{0}, \lambda_{1}\right)=\left(\frac{1}{\mu_{2}}, \frac{\mu_{1}}{\mu_{2}}\right)$ is an eigenvalue of the associated two-parameter system

$$
W_{i}(\boldsymbol{\lambda})=A_{i 0} \lambda_{0}+A_{i 1} \lambda_{1}+A_{i 2}, \quad i=1,2 .
$$

Here $A_{i j}$ are given by (5.4) and (5.5). We shall hereafter keep the notation $\boldsymbol{\mu}$ and $\boldsymbol{\lambda}$ as above, so if $\boldsymbol{\lambda}=\left(\lambda_{0}, \lambda_{1}\right)$ is an eigenvalue of (5.10), then $\boldsymbol{\mu}=\left(\mu_{1}, \mu_{2}\right)=\left(\frac{\lambda_{1}}{\lambda_{0}}, \frac{1}{\lambda_{0}}\right)$ is the corresponding eigenvalue of (5.1)-(5.3). Note that $\mu_{2}$ and $\lambda_{0}$ are always nonzero.

The eigenvalues of (5.1)-(5.3) form a countably infinite set of points without accumulation. There are only finitely many nonsemisimple eigenvalues and finitely many nonreal eigenvalues. Nonreal eigenvalues appear in conjugate pairs. See [8] Thms. 6.1 and 6.2] for these results.

The problem (5.1)-(5.3) for $i=1$ or $i=2$ has for a fixed value of $\boldsymbol{\mu}$ at most a one-dimensional space of solutions. If $\boldsymbol{\mu} \in \mathbb{R}^{2}$, then $W_{i}(\boldsymbol{\mu})$ is a selfadjoint operator. If $\boldsymbol{\mu} \in \mathbb{C}^{2} \backslash \mathbb{R}^{2}$, then $W_{i}(\boldsymbol{\mu})^{*}=W_{i}(\overline{\boldsymbol{\mu}})$, where ${ }^{-}$denotes complex conjugation. Since $W_{i}(\boldsymbol{\mu}) y_{i}=0$ holds if and only if $W_{i}(\overline{\boldsymbol{\mu}}) \bar{y}_{i}=0$, it follows that $\operatorname{dim} \mathcal{N}\left(W_{i}(\boldsymbol{\mu})\right)=$ $\operatorname{dim} \mathcal{N}\left(W_{i}(\boldsymbol{\mu})^{*}\right)=1$. We are now ready to establish Assumption III.

Lemma 5.4. Each eigenvalue $\boldsymbol{\mu}$ of the problem (5.1-5.3 is geometrically simple of finite ascent, and both $W_{i}(\boldsymbol{\lambda}), i=1,2$, are Fredholm operators of index 0 .

Proof. The remarks preceeding this lemma together with [16 Example III.5.14] establish everything except finite ascent, and the latter follows from [8, Thm. 6.6].

There are various differences between the approach of 8 and the one here. In 8] the eigenvalues $\boldsymbol{\mu}=\left(\mu_{1}, \mu_{2}\right)$ are considered. We use instead the eigenvalues $\boldsymbol{\lambda}=\left(\lambda_{0}, \lambda_{1}\right)$. In [8] root subspaces are considered only with respect to the pencil $\Delta_{2}-\mu_{2} \Delta_{0}$, while we consider joint root subspaces for pencils $\Delta_{i}-\lambda_{i} \Delta_{2}, i=0,1$. When bases for root subspaces are constructed our approach significantly reduces technical difficulties since all the geometric eigenspaces are now one-dimensional, i.e., all the joint eigenvalues are geometrically simple.

In order to be able to compare our results with those of [8] we need the following auxiliary results:

Lemma 5.5. If $\lambda_{0} \neq 0$ and $z_{1}, z_{2}, \ldots, z_{m} \in \mathcal{D}$ are such that

$$
\left(\Delta_{0}-\lambda_{0} \Delta_{2}\right) z_{j}=\Delta_{2} z_{j-1}, j=1,2, \ldots, m,
$$

where $z_{0}=0$, then

$$
u_{j}=\sum_{k=0}^{j-1}(-1)^{j}\left(\begin{array}{l}
j \\
k
\end{array}\right) \lambda_{0}^{j+k} z_{k+1}
$$

are such that

$$
\left(\Delta_{2}-\mu_{2} \Delta_{0}\right) u_{j}=\Delta_{0} u_{j-1}, j=1,2, \ldots, m,
$$

where $u_{0}=0$ and $\mu_{2}=\lambda_{0}^{-1}$. 
Proof. By induction on $j$ we prove that (5.11) implies

$$
\left(\Delta_{2}-\mu_{2} \Delta_{0}\right) z_{j}=\sum_{k=1}^{j-1}(-1)^{k} \mu_{2}^{k+1} \Delta_{0} z_{j-k}, \quad j=1,2, \ldots, m .
$$

Then (5.12) follows by a straightforward calculation which we omit.

We call a chain of vectors $u_{1}, u_{2}, \ldots, u_{m}$ such that (5.12) holds a Jordan chain for the pencil $\Delta_{2}-\mu_{2} \Delta_{0}$.

Corollary 5.6. Let $\mu_{2}=\lambda_{0}^{-1}$. Then $\mu_{2}$ is a semi-simple eigenvalue for the pencil $\Delta_{2}-\mu_{2} \Delta_{0}$ if and only if $\lambda_{0}$ is a semi-simple eigenvalue for $\Delta_{0}-\lambda_{0} \Delta_{2}$.

Lemma 5.7. Suppose that $\mathcal{R}_{m}$ is the $m$-th root subspace for (5.10) at an eigenvalue $\boldsymbol{\lambda}=\left(\lambda_{0}, \lambda_{1}\right)$ (see (1.7)). Then it follows that $\left(\Delta_{2}-\mu_{2} \Delta_{0}\right) \mathcal{R}_{m} \subset \Delta_{0} \mathcal{R}_{m-1}$. In particular, if $\mathcal{B}_{m}$ is the basis for $\mathcal{R}_{m}$ given in Theorem 4.1 then

$$
\begin{gathered}
\left(\Delta_{2}-\mu_{2} \Delta_{0}\right) z_{m j} \\
=\sum_{l=1}^{m-1}\left((-1)^{l} \mu_{2}^{l} \sum_{k=1}^{l} \sum_{g=1}^{d_{k}} \gamma_{j g}^{m k} \Delta_{0} z_{k g}\right),
\end{gathered}
$$

where $\gamma_{j g}^{m k}$ are defined inductively by

$$
\gamma_{j g}^{k+1, k}=c_{1 g j}^{2, k, k+1} \text { and } \gamma_{j g}^{k+r, k}=\sum_{l=k+1}^{k+r-1} \sum_{h=1}^{d_{l}} c_{1 h j}^{2, l, k+r} \gamma_{h g}^{l k}
$$

for $r=2,3, \ldots$

Proof. If we multiply the relation $\left(\Gamma_{0}-\lambda_{0} I\right) \mathcal{R}_{m} \subset \mathcal{R}_{m-1}$ by $-\lambda_{0}^{-1} \Delta_{2}$ on the left, we have that $\left(\Delta_{2}-\mu_{2} \Delta_{0}\right) \mathcal{R}_{m} \subset \Delta_{2} \mathcal{R}_{m-1}$. Then we prove by induction on $m$ that $\left(\Delta_{2}-\mu_{2} \Delta_{0}\right) \mathcal{R}_{m} \subset \Delta_{0} \mathcal{R}_{m-1}$. The second part of the lemma also follows by induction on $m$. We omit the details.

Now we compare our results with those of 8 . First we consider the case of a real eigenvalue. We assume that $\boldsymbol{\lambda} \in \mathbb{R}^{2}$ is an eigenvalue of (5.10), that $z_{1}=x_{11} \otimes x_{21}$ is a corresponding eigenvector and that

$$
S_{2}=\left[\begin{array}{ll}
\left\langle A_{11} x_{11}, x_{11}\right\rangle & \left\langle A_{11} x_{11}, x_{11}\right\rangle \\
\left\langle A_{20} x_{21}, x_{21}\right\rangle & \left\langle A_{21} x_{21}, x_{21}\right\rangle
\end{array}\right]
$$

Proposition 5.8. For $\boldsymbol{\lambda} \in \mathbb{R}^{2}$ the following are equivalent:

(i) $\boldsymbol{\lambda}$ is a semi-simple eigenvalue,

(ii) $\left\langle\Delta_{0} z_{1}, z_{1}\right\rangle \neq 0$,

(iii) the rank of the matrix $S_{2}$ is equal to 2 .

Proof. Conditions (i) and (ii) are equivalent by [8, Thm. 6.15] and Corollary 5.6 The equivalence of (i) and (iii) follows from [5. Thm. 6.3].

Proposition 5.9. For $\boldsymbol{\lambda} \in \mathbb{R}^{2}$ the following are equivalent:

(i) $\boldsymbol{\lambda}$ is not a semi-simple eigenvalue,

(ii) $\boldsymbol{\lambda}$ is a nonderogatory eigenvalue, but not algebraically simple,

(iii) $\left\langle\Delta_{0} z_{1}, z_{1}\right\rangle=0$,

(iv) the rank of the matrix $S_{2}$ is less than or equal to 1 . 
Proof. If rank $S_{2}$ is less than 2 , then it has to be equal to 1 . Namely, it follows from (iii') that $\left\langle A_{i 1} x_{i 1}, x_{i 1}\right\rangle \neq 0$ for $i=1,2$, and therefore $S_{2} \neq 0$. Then if $\boldsymbol{\lambda}$ is not semi-simple, it has to be nonderogatory. The equivalence of conditions (i)-(iv) now follows from Proposition 5.8 .

By the above propositions it follows that a basis $\mathcal{B}$ for a root subspace $\mathcal{R}$ at a real eigenvalue $\boldsymbol{\lambda}$ is described by Theorem 2.2. Then a root subspace $\mathcal{M}_{\mu_{2}}$ (in the notation of [8]) at a real eigenvalue $\mu_{2}$ is equal to the direct sum of the root subspaces corresponding to the eigenvalues $\boldsymbol{\lambda}=\left(\lambda_{0}, \lambda_{1}\right)$ with $\lambda_{0}=\frac{1}{\mu_{2}}$. Lemma 5.5 implies that a basis for $\mathcal{M}_{\mu_{2}}$ is thus obtained by Theorem 2.2 as the union of all the bases of the root subspaces $\mathcal{R}(\boldsymbol{\lambda})$ with $\lambda_{0}=\frac{1}{\mu_{2}}$. This yields Theorems 3.5 and 6.18 of [8] and also the Sturm-Liouville case of [3, Thm. 3.1]. The interested reader will find a numerical implementation of the algorithm for computation of a basis at a nonderogatory eigenvalue in 23.

Assume now that $\boldsymbol{\lambda}=\left(\lambda_{0}, \lambda_{1}\right)$ is a nonreal eigenvalue of (5.10). Then $\lambda_{0}$ is nonreal [8, p. 99]. We write $z_{1}=x_{11} \otimes x_{21}$ for an eigenvector correponding to $\boldsymbol{\lambda}$ and

$$
S_{2}=\left[\begin{array}{ll}
\left\langle A_{10} x_{11}, \bar{x}_{11}\right\rangle & \left\langle A_{11} x_{11}, \bar{x}_{11}\right\rangle \\
\left\langle A_{20} x_{21}, \bar{x}_{21}\right\rangle & \left\langle A_{21} x_{21}, \bar{x}_{21}\right\rangle
\end{array}\right] \text {. }
$$

Proposition 5.10. For a nonreal eigenvalue $\boldsymbol{\lambda}$ the following are equivalent:

(i) $\boldsymbol{\lambda}$ is semi-simple,

(ii) $\left\langle\Delta_{0} z_{1}, \bar{z}_{1}\right\rangle \neq 0$,

(iii) the rank of the matrix $S_{2}$ is equal to 2 .

Proof. Conditions (i) and (ii) are equivalent by [8, Thm. 6.14] and Corollary 5.6 The equivalence of (i) and (iii) follows from [5. Thm. 6.3].

If $\boldsymbol{\lambda}$ is not semi-simple and $\operatorname{rank} S_{2}$ is equal to 1 , then $\boldsymbol{\lambda}$ is nonderogatory and Theorem 2.2 gives a basis for the corresponding root subspace. By Lemma 5.7 it follows that the union of bases, of root subspace at the eigenvalues $\boldsymbol{\lambda}$ for which $\lambda_{0}=\frac{1}{\mu_{2}}$, is a basis for the root subspace $\mathcal{M}_{\mu_{2}}$ (in notation of [8]). If $\mathcal{M}_{\mu_{2}}$ is a root subspace at a nonreal eigenvalue $\mu_{2}$, it is equal to the direct sum of the root subspaces corresponding to the eigenvalues $\boldsymbol{\lambda}=\left(\lambda_{0}, \lambda_{1}\right)$ with $\lambda_{0}=\frac{1}{\mu_{2}}$. If all these eigenvalues $\boldsymbol{\lambda}$ are nonderogatory, then Lemma 5.5 implies that a basis for $\mathcal{M}_{\mu_{2}}$ is obtained by Theorem 2.2. This yields Theorems 6.9 and 6.19 of [8] and it also gives a basis for those $j$ in [8, pp. 118-122] for which $s_{2}<j \leq s_{1}$. The eigenvalues $\boldsymbol{\lambda}$ that correspond to the remaining $j$ (i.e., such that $1 \leq j \leq s_{2}$ ) are derogatory, i.e., $S_{2}=0$. Now we use Theorem 4.1 to obtain a basis $\mathcal{B}$ for the root subspace at $\boldsymbol{\lambda}$. The main result about bases for $\mathcal{M}_{\mu_{2}}$ in 8 is Theorem 6.20. The basis that Faierman gives there is a union of bases of root subspaces $\mathcal{R}(\boldsymbol{\lambda})$ over all $\boldsymbol{\lambda}$ with $\lambda_{0}=\frac{1}{\mu_{2}}$. These are of two types: for $s_{2}<j \leq s_{1}$ the eigenvalues $\boldsymbol{\lambda}$ are nonderogatory and our Theorem 2.2 gives the required properties described in 8 . Lemma 6.9]. The other eigenvalues with $1 \leq j<s_{2}$ are derogatory. Bases for $\mathcal{R}(\boldsymbol{\lambda})$ are described in Conjecture 6.1 of [8], which is established in [8] only when the ascent of $\Gamma_{0}$ is at most 3. Our basis in Theorem 4.1 is constructed in all cases of finite ascent. We hope to discuss the precise relationship with [8 Conj. 6.1] in a separate publication.

Finally, we remark that bases for $\mathcal{R}(\boldsymbol{\lambda})$ can be used to obtain completeness and expansion results as carried out in [8, Thms. 6.7 and 6.8$]$ and $[\underline{8}, \S 6.7]$, respectively. 
Then orthonormal bases are important. These can be constructed by a GramSchmidt type orthogonalization applied inductively for each $m$ in the construction of a basis for $\mathcal{R}(\boldsymbol{\lambda})$. Additional care needs to be taken for the root subspaces at pairs of conjugate eigenvalues; for other pairs of eigenvalues the root subspaces are orthogonal by [8, Thm. 6.1].

\section{REFERENCES}

[1] F.V. Atkinson. Multiparameter Eigenvalue Problems. Academic Press, 1972. MR 56:9291

[2] P.A. Binding. Left Definite Multiparameter Eigenvalue Problems. Trans. Amer. Math. Soc., 272:475-486, 1982. MR 83h:47015

[3] P.A. Binding. Multiparameter Root Vectors. Proc. Edin. Math. Soc., 32:19-29, 1989. MR 90a:47048

[4] P.A. Binding and P.J. Browne. Asymptotics of Eigencurves for Second Order Ordinary Differential Equations I. J. Diff. Eq., 88:30-45, 1990. MR 92b:34101

[5] P.A. Binding and T. Košir. Second Root Vectors for Multiparameter Eigenvalue Problems of Fredholm Type. Trans. Amer. Math. Soc., 348:229-249, 1996. MR 96f:35121

[6] P.A. Binding and K. Seddighi. Elliptic Multiparameter Eigenvalue Problems. Proc. Edin. Math. Soc., 30:215-228, 1987. MR 88g:47036

[7] G. Birkhoff. Lattice Theory, volume 25 of Amer. Math. Soc. Colloq. Publ., 3rd edition, Amer. Math. Soc., Providence, 1973. MR 37:2638

[8] M. Faierman. Two-parameter Eigenvalue Problems in Ordinary Differential Equations, volume 205 of Pitman Research Notes in Mathematics. Longman Scientific and Technical, Harlow, U.K., 1991. MR 93b:47095

[9] G.A. Gadzhiev. Introduction to Multiparameter Spectral Theory (in Russian), Azerbaijan State University, Baku, 1987.

[10] I.C. Gohberg, P. Lancaster, and L. Rodman. Invariant Subspaces of Matrices with Applications. Wiley-Interscience, 1986. MR 88a:15001

[11] L. Grunenfelder and T. Košir. An Algebraic Approach to Multiparameter Eigenvalue Problems. Trans. Amer. Math. Soc., 348:2983-2998, 1996. MR 96j:47015

[12] L. Grunenfelder and T. Košir. Coalgebras and Spectral Theory in One and Several Parameters. In the series Operator Theory: Adv. and Appl., 87: 177-192, Birkhäuser-Verlag, 1996. MR 97k:47019

[13] L. Grunenfelder and T. Košir. Geometric Aspects of Multiparameter Spectral Theory, Trans. Amer. Math. Soc., 350:2525-2546, 1998. MR 98h:13032

[14] H.(G.A.) Isaev. Lectures on Multiparameter Spectral Theory. Dept. of Math. and Stats., University of Calgary, 1985.

[15] G.A. Isaev and A.S. Fainshtein. The Taylor Spectrum and Multiparameter Spectral Theory for Systems of Operators. Soviet Math. Dokl., 36:413-417, 1988. MR 88m:47028

[16] T. Kato. Perturbation Theory for Linear Operators, volume 132 of Grundlehren der math. Wiss. Springer-Verlag, second edition, 1984. MR 53:11389

[17] T. Košir. Commuting Matrices and Multiparameter Eigenvalue Problems. PhD thesis, Dept. of Math. and Stats., University of Calgary, 1993.

[18] T. Košir. On the Structure of Commutative Matrices. Lin. Alg. Appl., 187:163-182, 1993. MR 94i:15011

[19] T. Košir. The Finite-Dimensional Multiparameter Spectral Theory: The Nonderogatory Case. Lin. Alg. Appl., 212/213:45-70, 1994. MR 95k:15020

[20] T. Košir. On the Structure of Commutative Matrices II. Lin. Alg. Appl., 261:293-305, 1997. MR 98k:15018

[21] T. Košir. Root Vectors for Geometrically Simple Multiparameter Eigenvalues, to appear in Int. Equat. Oper. Theory.

[22] J. Meixner, F. W. Schäfke, and G. Wolf. Mathieu Functions and Spheroidal Functions and Their Mathematical Foundations, volume 873 of Lect. Notes in Math., Springer-Verlag, 1980. MR 83b:33013

[23] B. Plestenjak. A Numerical Algorithm for Computing a Basis for the Root Subspace at a Nonderogatory Eigenvalue of a Multiparameter System, Lin. Alg. Appl., 285:257-276, 1998. MR 2000a:65048 
[24] B.D. Sleeman. Multiparameter Spectral Theory in Hilbert Space, volume 22 of Pitman Research Notes in Mathematics. Pitman Publ. Ltd., London U.K., Belmont U.S.A., 1978. MR 81h:47004

[25] A.E. Taylor and D.C. Lay. Introduction to Functional Analysis. Wiley, New York, second edition, 1980. MR 81b:46001

[26] H. Volkmer. Multiparameter Eigenvalue Problems and Expansion Theorems, volume 1356 of Lecture Notes in Mathematics. Springer-Verlag, Berlin, New York, 1988. MR 90d:47021

Department of Mathematics and Statistics, University of Calgary, Calgary, AlBERTA, CANADA T2N 1N4

E-mail address: binding@ucalgary.ca

Department of Mathematics, University of Ljubljana, Jadranska 19, 1000 Ljubljana, Slovenia

E-mail address: tomaz.kosir@fmf.uni-lj.si 